\title{
A GENETIC ALGORITHM FOR THE STEEL CONTINUOUS CASTING WITH INTER-SEQUENCE DEPENDENT SETUPS AND DEDICATED MACHINES
}

\author{
Abdelkader Sbihi $^{1, *}$ and Makram Chemangui ${ }^{2}$
}

\begin{abstract}
The steel continuous casting planning and scheduling problem namely SCC is a particular hybrid (flexible) flowshop that includes stages: (i) the converters (CV), (ii) the refining stands (RS) and (iii) the continuous casting (CC) stages. In this paper we study the SCC with inter-sequence dependent setups and dedicated machines at the last stage. The batch sequences are assumed to be pre-determined for one of the $\mathrm{CC}$ devices with a non preemptive scheduling process. The aim is to schedule the batches for each CC machine including the times setup between two successive sequences. We model the problem as a MILP where the objective is to minimize the makespan $C_{\max }$ that we formulate as the largest completion time taking account of the setup times for each CC. Then, we propose an adapted genetic algorithm that we call Regeneration GA (RGA) to solve the problem. We use a randomly generated instances of several sizes to test the model and for which we do not know an optimal solution. The method is able to solve the problems in an acceptable time for medium and large instances while a commercial solver was able to solve only small size instances.
\end{abstract}

Mathematics Subject Classification. 90B35, 90B50, 90C11, 90C59

Received May 4, 2017. Accepted March 11, 2018.

\section{INTRODUCTION}

The steel continuous casting problem (SCC) is a particular scheduling problem that arises from the steel making industry. The problem comprises three main stages that are the converters (CV), the refining stands (RS) and the continuous castings (CC) where each stage includes one or more parallel devices. Each charge is to be process on one device at each stage while each device has to process only one charge separately. With this configuration (Figs. 1 and 2), the SCC can be seen as a hybrid flowshop (HFS) (or flexible flowshop (FFS)) problem (see [14, 28]). However, the difference with HFS resides in the continuous processing (non preemptive process) of the charges while taking into account the inter-sequence dependent setup times at the last stage $(\mathrm{CC})$.

To show the difference between the HFS and the SCC, we make in the following a comparative study.

For a manufacturing system, the production is organized with regards to a given number of operations or stages to be accomplished with respect to the same order. Hence, the devices are supposed to carry setup

Keywords and phrases: SCC, GA, scheduling, $C_{\max }$, setup.

1 Université Le Havre Normandie, LMAH, FR CNRS 3335, ISCN, 76600 Le Havre, France.

2 Audencia Business School, 8 route de la Jonelière, 44312 Nantes, France.

* Corresponding author: abdelkader.sbihi@univ-paris1.fr 


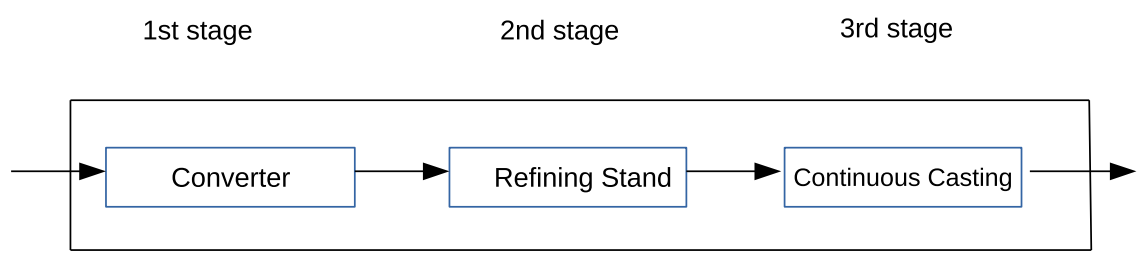

FiguRe 1. A generic SCC configuration.

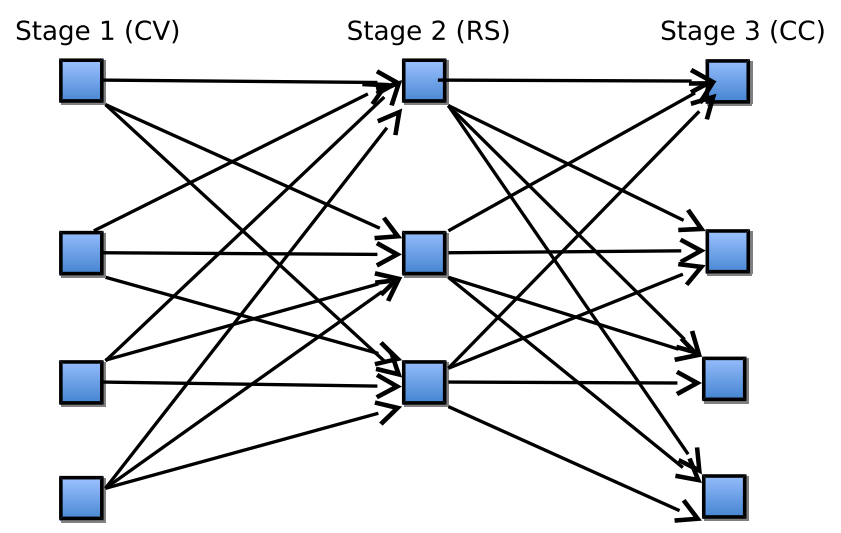

Figure 2. A $\left(\left(\mathrm{P}_{i}\right)_{i=1}^{m_{1}},\left(\mathrm{Q}_{j}\right)_{j=1}^{m_{2}},\left(\mathrm{R}_{k}\right)_{k=1}^{m_{3}} \| \sigma \mid C_{\max }\right)$ configuration.

times and two different cases are considered: (i) asynchronous transfer of the product flow from a current stand to the next one, (ii) and synchronized transfer of the product flow from one device to another. In general, the asynchronous transfer case is called a flowshop. The classical flowshop problem (FSP) considers only one machine at each stage, while its generalization considers a set of devices in series with multicapacity in parallel, called a hybrid flowshop problem (HFS). On one hand, the hybrid flowshop problem (HFS) can be seen as a set of parallel machines in series. On the other hand, the SCC planning and scheduling problem is a complex hybrid flowshop (HFS) scheduling problem with the added properties: (i) job grouping in sequences, (ii) precedence constraints, (iii) no preemptive scheduling process for the same sequence of charges, (iv) and setup time constraints on the last stage (CC stage).

More generally, the objective of the SCC is to determine the sequences order, the schedules and the system of devices assignments for the entire production processes. Moreover, we may notice that the SCC problem is known in the literature as a hard scheduling problems (see $[11,34,36])$.

In the following, we represent the SCC problem as a 3 stages scheduling problem with $m_{r}(r=1, \ldots, 3)$ parallel machines at each stage $r$ :

(1) $m_{1}$ identical parallel machines $\mathrm{CV}$ at the 1st stage (noted $\left.\left(\mathrm{P}_{i}\right)_{i=1}^{m_{1}}\right)$,

(2) $m_{2}$ uniform parallel machines RS at the 2nd stage (noted $\left(\mathrm{Q}_{j}\right)_{j=1}^{m_{2}}$ ),

(3) $m_{3}$ unrelated parallel machines $\mathrm{CC}$ at the 3 rd stage (noted $\left.\left(\mathrm{R}_{k}\right)_{k=1}^{m_{3}}\right)$.

Similarly to the notation by [7], we write the SCC problem as a 3-stages hybrid flowshop (HFS): $\left(\mathrm{P}_{i}\right)_{i=1}^{m_{1}},\left(\mathrm{Q}_{j}\right)_{j=1}^{m_{2}},\left(\mathrm{R}_{k}\right)_{k=1}^{m_{3}} \| \sigma \mid C_{\max }$, where $\|$ stands for a parallel system and $\sigma$ is the inter-sequence setup time (Fig. 2).

At the last stage the system includes the setup times that represent the inter-sequence times necessary to a $\mathrm{CC}$ machine between the processing termination of the current sequence and the processing start of the next sequence (between the last charge of the current sequence and the first charge of the next sequence) such that: 
- the sojourn (transit) time is minimized;

- the due date is met;

- the continuity constraints are satisfied;

- no machine breakdown is considered.

The scheduling problems are mostly NP-hard optimization problems (see [5, 13]) and in the papers by [22], the authors showed that HFS problems with the objective to minimize the makespan are NP-complete. Also, the $m$-stages HFS with unrelated parallel machines and dependent setup times has been proven to be NP-hard (see $[14,16])$. Also, there exist many variants of the SCC problem (see [25, 29, 37]) which they differ by the objective to optimize and/or by the set of constraints to handle. In many literature works, the setup time is considered as a constant or a parameter given in an interval (see $[3,31])$.

In this paper we propose an MILP to model the SCC production problem. The objective function includes the setup time as a decision variable which contributes to minimize the makespan by reducing the gap between sequences on the same CC machine at the last stage. Also, we consider the makespan to minimize as the largest completion time for all the CC machines. Moreover, we recall that the proposed problem has a very complex structure, where small size problems could be difficult to solve to their optimum in a reasonable run time.

To overcome this difficulty, we intend to develop an approximate approach to solve the SCC. The novelty of the proposed planning and scheduling approach resides in optimizing the casting sequencing and the steelmaking scheduling simultaneously. The Regeneration GA is designed with specific components based on the schedule optimization search. We, first, compare the numerical experiments obtained by the standard GA with those obtained by the RGA approach. This enables us to claim the high efficiency of RGA regarding the standard GA. We used the quality of the solution and the consuming time by both GA and RGA. Then, we set the algorithm parameters to their best settings to test a set of instances of different sizes.

In the remainder, the paper is organized as follows. In Section 2, we give a literature survey on the hybrid (flexible) flowshop and the steel-making scheduling problems. We recall several exact and approximate approaches that have been developed for such problems. Section 3 describes the problem and details its structure. Section 4 presents the SCC problem, formulates the makespan $C_{\max }$ and details the mathematical formulation as a MILP subject to a set of constraints where the objective is to minimize $C_{\max }$. In Section 5 , we develop a genetic based approach to approximately solve the SCC problem. We explain the start time computation for each charge at the first stage (CV) and detail the procedures used to compute an efficient solution. We also explain and discuss the main steps of the RGA approach and the auxiliary procedures. In Section 6, we detail and discuss the numerical experiments by showing the efficiency of our approach. Finally in Section 7, we give a conclusion that summarizes the study and shows some potential research perspectives to improve the current results.

\section{LITERATURE SURVEY AND PROBLEM POSITIONING}

Numerous research works have been developed to solve several HFS and SCC variants. In this section, we review the most known approaches (exact and approximate) that have been developed in previous research works to address the HFS in general and the SCC in particular. In the paper by [36], the authors reviewed and proposed a classification of several SCC models. In [2, 11], the authors detailed both mathematical and non-mathematical techniques used to solve these variants.

Moreover, optimization methods such as local search, genetic algorithms (see [42]), swarm intelligence optimization (see [12]) or mathematical programming (see [3, 31]) have been developed to solve the SCC problem. In the papers by $[8,24,43]$ several types of metaheuristics have been applied to the SCC problem. Hybrid approaches have also been tailored for a continuous steel plant (see $[1,4,10])$.

We also may recall that simulation techniques (see [44]) have been applied to simulate the production environment and efficient solutions have been computed for such problems. In [9], the authors have designed a multi-agent based system to evaluate a dynamic casting schedule. Other techniques such as graphs theory and mathematical programming approaches have also been used. For instance, in [20], the authors have developed 
a continuous slab caster schedule using interval graphs class. In the paper by [17], the authors have applied a multiple knapsack problem based ILP to avoid distortion between the surplus inventory and orders.

In [35], the authors have used a general heuristic based Lagrangean relaxation and dynamic programming for a non-linear programming model to address the machines conflicts. In [15], the authors developed a decomposition method of the MILP to solve large size scheduling problems. One can cite the paper by [37] in which the authors have relaxed the capacity constraints, then applied a dynamic programming for solving the relaxed problem. In the paper by [40], an approach based on the production control has been developed to identify the best steelmaking operations level. The paper by [38] proposed another approach to minimize the total weighted termination time by relaxing the precedence constraints.

In [26], the SCC problem has been solved by a beam search method. Tabu search (see [39]) has been applied for the SCC to schedule the production in order to maximize the production quality while minimizing the costs and other penalties. In [41], the authors presented an approximate approach to minimize the completion time based on batch decoupling. Also, in [25], the authors developed a Lagrangean relaxation coupled with cuts generation to compute better bounds for the relaxed capacity constraints problem. Particular SCC problems with different configurations have also been solved. For instance, in the article by [23], the authors have developed a 3-stages heuristic procedure to schedule jobs for a complex steel plant with $3 \mathrm{CV}, 2 \mathrm{RS}$ and $4 \mathrm{CC}$ to improve the schedule by using an LP model.

Also several evolutionary based algorithms have been applied to the SCC. One can cite the paper by [19] in which an evolutionary algorithms has been developed for a two-strands CC scheduling system. In the paper by [6], the authors proposed a GA to identify the efficient solutions in the Pareto front after running some extensive search. In the paper by [33], the authors have developed a GA they called hybrid constructive genetic algorithm (HCGA) to solve the HFS. Their approach used two fitness functions (total cost and local search optimization) to evaluate an individual. A multi-objective GA to minimize the makespan and tardiness penalties has been developed by [18]. Recently, in [21], the authors have presented a self-adaptive GA to optimize the casts in order to minimize the makespan and the total idle times.

During the last two decades, other evolutionary approaches such as ant colony (ACO), bee colony (BCO) or swarm optimization (SO) have also been applied for the SCC. In the article by [1], one can find an approach based on the combination of ACO and nonlinear optimization methods. In [28], the authors have developed a $\mathrm{BCO}$ heuristic. In the paper by [27], the author has proposed a cooperative co-evolutionary artificial $\mathrm{BCO}$ algorithm with two sub-swarms to solve the SCC by using some self-adaptive neighborhood operators.

Due to the NP-hardness of SCC, we develop an adapted genetic algorithm that we call Regeneration GA (RGA) based heuristic to solve the SCC. The choice of the genetic based optimization is efficient since this algorithm fits well with the structure of the SCC problem. In particular, coding the chromosome fits well with the solution structure of the problem to assign the charges to each CV, RS or CC machine while ensuring the validity of the individual. The proposed approach solves the problem by optimizing the order and the schedules with inter-sequence dependent setup times on each dedicated CC device. The approach combines: (i) the planning of the casting of the batches and (ii) the steelmaking scheduling of the total charges. These two components are integrated in the model. In the next sections, we describe the GA features by detailing the used genetic operators. We also present the approach performance obtained by the experiments on a random generated set of instances inspired by a real production situation.

\section{Problem statement}

In the SCC problem $\equiv \mathrm{P}_{m_{1}}, \mathrm{Q}_{m_{2}}, \mathrm{R}_{M m_{3}} \| \sigma \mid C_{\max }$, the production orders are represented by a set of the cast sequences (batch) where each sequence is dedicated to a CC machine at the last stage. At the first stage, the charges can be processed individually on any one of the available converters $(\mathrm{CV})$. Then they are transferred to the first available refining stand (RS). However, at the stage 3, the processing of the charges on the CC is predetermined. That means that the actual allocation of charges and sequence of charges on each CC line is predetermined and only the timing of the charges needs to be established. 


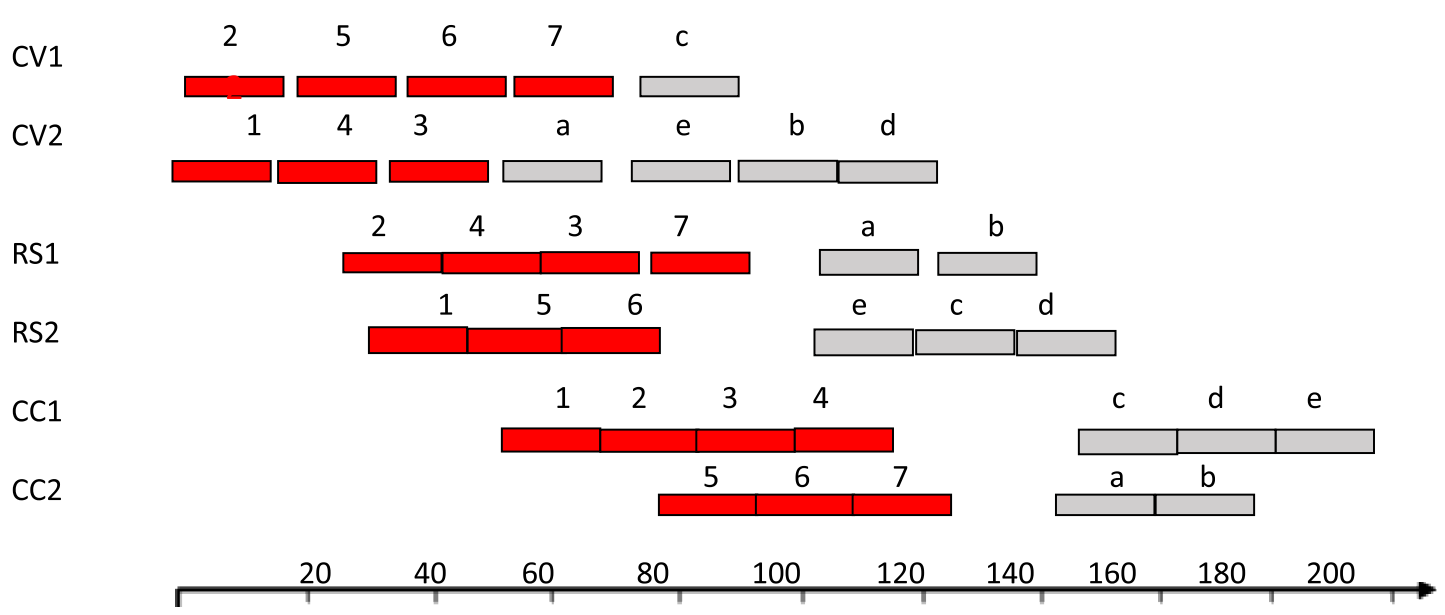

Figure 3. A 2 CV-2 RS-2 CC Gantt chart.

We assume that the sojourn (transit) time for the charges between their termination at the first stage and their start at the last stage $(\mathrm{CC})$ is limited. The processing time for each charge at the last stage is a bounded value. Also, we add an availability date for each converter so that the start time at the first stage is different for all the converters. We set the objective as to maximize the productivity by minimizing the $C_{\max }$.

\subsection{Process, sequences and continuity constraints}

The planning system is detailed in the following:

- a sequence is a set of charges dedicated to a $\mathrm{CC}$ with precedence constraints for the charges;

- any converter $\mathrm{CV}$ can be used in the first stage with a constant processing time;

- the charge has a limited sojourn time between the termination in the CV and the start at CC;

- there is no idle time for the charges on the CC due to the continuous constraints;

- the processing times at the CC stage are decision variables belonging to a given interval;

- the sequence start times at CC are bounded and the waiting time between two successive sequences represents the setup time.

The example in Figure 3 shows the sequencing of a batch composed of 4 sequences on a (2CV-2RS-2CC) system. The first batch is composed of 2 sequences noted $(1,2,3,4) \times(c, d, e)$ with respectively 4 and 3 charges which are dedicated to $\mathrm{CC}_{1}$, while the second batch is composed of 2 sequences noted $(5,6,7) \times(a, b)$ with respectively 3 and 2 charges and are dedicated to $\mathrm{CC}_{2}$. Between $\mathrm{CV}$ and $\mathrm{RS}$ stages the charges are processed individually, while the sequences are re-assembled to be processed on their assigned CC. For example, the charge noted " $a$ " is belonging to the second sequence and is to be processed on $\mathrm{CC}_{2}$. It starts at time 50 on $\mathrm{CV}_{2}$, is transferred to $\mathrm{RS}_{1}$ at time 101 then it is transferred to $\mathrm{CC}_{2}$ and starts at time 142 .

\section{Continuous Casting of the Pre-Ordered Sequences}

In this section we give a mathematical formulation for the SCC problem taking into account the set of constraints and the objective function to optimize. The obtained MILP aims at minimizing the makespan. The production environment is a deterministic one and the number of charges together with their intrinsic data are assumed to be known in advance. Also, we assume that the number of parallel machines at each stage is known in advance. 


\subsection{Notations}

To better understand the problem, we detail the used notations and all the data in the next sections.

\subsubsection{Sets, constants and indices}

(1) $(r=1,2,3)$ denote the stages (CV,RS,CC);

(2) $m_{r}:$ number of machines at the stage $r$;

(3) $i=1, \ldots, m_{1}$ (resp. $\left.j=1, \ldots, m_{2}\right)$ (resp. $\left.k=1, \ldots, m_{3}\right)$ denotes the index of a machine at the 1 st stage (resp. the 2 nd stage) (resp. the 3 rd stage) such that $\left(\mathrm{CV}_{i}, \mathrm{RS}_{j}, \mathrm{CC}_{k}\right)$ is a production line;

(4) $S_{k}$ : number of the sequences $s_{k}$ to process on the machine $\mathrm{CC}_{k}\left(s_{k}=1, \ldots, S_{k}\right)$;

(5) $s_{k}$ : a sequence to process on $\mathrm{CC}_{k}\left(k=1, \ldots, m_{3}\right)$;

(6) $n_{s_{k}}=\left|s_{k}\right|$ : number of the pre-ordered charges (jobs) $c$ for a sequence $s_{k}$ on $\mathrm{CC}_{k}$;

(7) $n_{k}=\sum_{s_{k}=1}^{S_{k}} n_{s_{k}}$ : total number of charges to process on $\mathrm{CC}_{k}\left(k=1, \ldots, m_{3}\right)$;

(8) $c$ : index of a charge of the sequence $s_{k}$ dedicated to $\mathrm{CC}_{k}\left(c=1, \ldots, n_{k}\right)$;

(9) $N=\sum_{k=1}^{m_{3}} n_{k}$ : total number of charges in the system.

We also have the following assumptions:

(1) $\pi_{i}$ : position of a charge on the first $\left(m_{1}-1\right) \mathrm{CV}_{i}\left(\pi_{i}=1, \ldots, \Pi_{i}\right.$; $\left.i=1, \ldots, m_{1}-1\right)$ where $\Pi_{i}=\left\lfloor\frac{\sum_{k=1}^{m_{3}} n_{k}+1}{m_{1}}\right\rfloor$;

(2) $\pi_{m_{1}}$ : position of a charge on the last machine $\mathrm{CV}_{m_{1}}\left(\pi_{m_{1}}=1, \ldots, \Pi_{m_{1}}\right)$ where $\Pi_{m_{1}}=\left\lfloor\frac{\sum_{k=1}^{m_{3}} n_{k}}{m_{1}}\right\rfloor$.

\subsubsection{Data, parameters and settings}

(1) $p^{1}$ : processing time of a charge $c\left(c=1, \ldots, n_{k}\right)$ on the converters $\mathrm{CV}_{i}\left(i=1, \ldots, m_{1}\right)$ at the 1 st stage;

(2) $p_{j}^{2}$ : processing time of a charge $c$ on the refining stand $\mathrm{RS}_{j}\left(j=1, \ldots, m_{2}\right)$ at the 2 nd stage;

(3) $\left[P_{k, c}^{\min }, P_{k, c}^{\max }\right]$ : interval of the processing time $p_{k, c}^{3}$ of a charge $c$ dedicated to $\mathrm{CC}_{k}$;

(4) $\left[\sigma_{s_{k}}^{\min }, \sigma_{s_{k}}^{\max }\right]$ : interval of $\sigma_{s_{k}}$, the inter-sequence dependent setup time between sequences $s_{k}$ and $\left(s_{k}+1\right)$ $\left(s_{k}=1, \ldots, S_{k}-1\right)$

(5) $d_{i}$ : availability date for $\mathrm{CV}_{i}$;

(6) $T_{k, c}$ : maximum allowed sojourn time for a charge $c$ between the processing termination in any $\mathrm{CV}_{i}$ and its start in $\mathrm{CC}_{k}$;

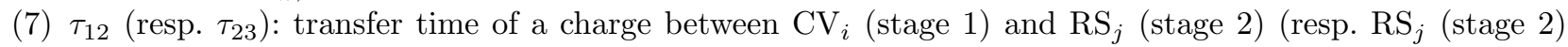
and $\mathrm{CC}_{k}$ (stage 3$\left.)\right)$.

(8) $d_{k, c}$ : due date for a charge $c$ dedicated to $\mathrm{CC}_{k}$

\subsubsection{Decision variables}

We have identified both continuous and binary decision variables for the proposed model. For any $\mathrm{CC}_{k}$ $k=1, \ldots, m_{3}$ :

(1) $x_{k, c}^{r}$ : start time of the charge $c\left(c=1, \ldots, n_{k}\right)$ dedicated to $\mathrm{CC}_{k}$ at stage $r(r=1,2,3)$;

(2) $p_{k, c}^{3}$ : processing time of charge $c\left(c=1, \ldots, n_{k}\right)$ dedicated to $\mathrm{CC}_{k}$ at the $3^{r d}$ stage;

(3) $\sigma_{s_{k}}$ : setup time between two successive sequences $s_{k}$ and $\left(s_{k}+1\right)$ to process at $\mathrm{CC}_{k}$ (between the last charge $n_{s_{k}}$ of $s_{k}$ and the first charge $1_{\left(s_{k}+1\right)}$ of $\left.s_{k+1}\right)$ for $s_{k}=1, \ldots, S_{k}-1$. 
(4)

$$
\begin{aligned}
& y_{k, c}^{\pi_{i}}= \begin{cases}1 & \text { if the dedicated charge } c \text { to } \mathrm{CC}_{k} \text { is assigned to a position } \pi_{i} \text { in } \mathrm{CV}_{i}, \\
0 & \text { otherwise. }\end{cases} \\
& c=1, \ldots, n_{k}, \pi_{i}=1, \ldots, \Pi_{i}, i=1, \ldots, m_{1} .
\end{aligned}
$$

\subsection{A SCC mathematical model}

The model computes the processing start time of the charge defined by its position at the first stage (CV) regarding the precedence constraint. It also respects the charge assignment to the CC machine.

\subsubsection{Constraints}

For each $\mathrm{CC}_{k}\left(k=1, \ldots, m_{3}\right)$, the constraints are formulated as follows:

$$
\begin{aligned}
& \sum_{i=1}^{m_{1}} \sum_{\pi_{i}=1}^{\Pi_{i}} y_{k, c}^{\pi_{i}}=1 ; \quad c=1, \ldots, n_{k} \\
& \sum_{k=1}^{m_{3}} \sum_{c=1}^{n_{k}} y_{k, c}^{\pi_{i}}=1 ; \quad \pi_{i}=1, \ldots, \Pi_{i}
\end{aligned}
$$

and $\quad \sum_{k=1}^{m_{3}} y_{1,1}^{1}=1$

$$
\begin{aligned}
y_{k, c+1}^{t_{i}+1} & \leq \sum_{i=1}^{m_{1}-1} \sum_{\pi_{i}=1}^{t_{i}} y_{k, c}^{\pi_{i}} ; \quad c=1, \ldots, n_{k}-1 ; t_{i}=1, \ldots, \Pi_{i}-1 ; \\
y_{k, c+1}^{t_{m_{1}}} \leq & \leq \sum_{i=1}^{m_{1}} \sum_{\pi_{i}=1}^{t_{i}} y_{k, c}^{\pi_{i}}-y_{k, c}^{t_{m_{1}}} ; \quad c=1, \ldots, n_{k}-1 ; t_{i}=1, \ldots, \Pi_{i} ; \\
x_{k, c}^{1} & =\sum_{i=1}^{m_{1}} \sum_{\pi_{i}=1}^{\Pi_{i}}\left[d_{i}+p^{1}\left(\pi_{i}-1\right)\right] y_{k, c}^{\pi_{i}} ; \quad c=1, \ldots, n_{k}
\end{aligned}
$$

Refining stage

$$
\begin{aligned}
x_{k, c+1}^{2} & \geq x_{k, c}^{2}+p_{j}^{2} ; \quad c=1, \ldots, n_{k}-1, j=1, \ldots, m_{2} ; \\
x_{k, c}^{2} & \geq x_{k, c}^{1}+p^{1}+\tau_{12} ; \quad c=1, \ldots, n_{k}
\end{aligned}
$$

Continuous casting stage

$$
\begin{aligned}
& x_{k, c}^{3} \geq x_{k, c}^{2}+p_{j}^{2}+\tau_{23} ; c=1, \ldots, n_{k}, j=1, \ldots, m_{2} ; \\
& x_{k, c+1}^{3}=x_{k, c}^{3}+p_{k, c}^{3} ; \forall c \notin\left\{n_{1}, n_{1}+n_{2}, \ldots, \sum_{s_{k}=1}^{S_{k}} n_{s_{k}}\right\}, \forall k=1, \ldots, m_{3} ; \\
& x_{k, c+1}^{3} \geq x_{k, c}^{3}+p_{k, c}^{3}+\sigma_{\ell} ; \forall c=\sum_{s_{k}=1}^{\ell} n_{s_{k}}, \ell=1, \ldots, S_{k}-1 ;
\end{aligned}
$$




$$
\begin{aligned}
& p_{k, c}^{3} \in\left[P_{k, c}^{\min }, P_{k, c}^{\max }\right] ; c=1, \ldots, n_{k} ; \\
& x_{k, c}^{3}-\left(x_{k, c}^{1}+p^{1}\right) \leq T_{k, c}, c c=1, \ldots, n_{k} ; \\
& \sigma_{s_{k}} \in\left[\sigma_{s_{k}}^{\min }, \sigma_{s_{k}}^{\max }\right] ; s_{k}=1, \ldots, S_{k}-1 ; \\
& x_{k, c}^{3}+p_{k, c}^{3} \leq d_{k, c} ; c=1, \ldots, n_{k} .
\end{aligned}
$$

Constraint (4.1) means that a charge $c\left(c=1, \ldots, n_{k}\right)$ is assigned to one and only one machine $\mathrm{CV}_{i}$ and at one and only one position $\pi_{i}$.

Constraint (4.2) means that a converter $\mathrm{CV}_{i}$ has to process one and only one charge $c$ at a specific position $\pi_{i}\left(i=1, \ldots, m_{1}\right)$. Also, only the charge $c=1$ dedicated to $\mathrm{CC}_{1}$ has to be assigned to the first position at the converter $\mathrm{CV}_{1}$.

Constraint (4.3) requires that the charge $(c+1)$ has to be processed on the converter $\mathrm{CV}_{1}(i=1)$ at the position $\left(t_{1}+1\right)$ only if the charge $c$ is to process on one of the $\left(m_{1}-1\right)$ first converters $\mathrm{CV}_{i}$ and at any positions between 1 and $t_{i}$.

Similarly to the constraint above, constraint (4.4) means the same in the case of the last converter $\mathrm{CV}_{m_{1}}$ for two successive charges $c$ and $(c+1)$.

Constraint (4.5) stands for the start time to process the charges in the first stage $\mathrm{CV}_{i}\left(i=1, \ldots, m_{1}\right)$;

Constraint (4.6) means that for two successive charges to process on the same refining stand $\left(\mathrm{RS}_{j}\right)$, the second charge can be processed only if the first one has reached its end time.

Constraints (4.7) and (4.8) require that for two successive operations for the same charge, the second operation can only start once the first one has reached its end time and the charge has been transferred to the next stage.

Moreover, constraints (4.7) (resp. constraint (4.8)) stands for the precedence rules between $\mathrm{CV}_{i}$ and $\mathrm{RS}_{j}$ (resp. between $\mathrm{RS}_{j}$ and $\mathrm{CC}_{k}$ ).

Constraint (4.9) stands for the continuity constraints between charges $c$ and $(c+1)$ for a given sequence $s_{k}$ (no preemptive process).

Constraint (4.10) stands for inter-sequence dependent setup times between two successive sequences (i.e. between the last charge of the current sequence and the first charge of the next sequence) for the same $\mathrm{CC}_{k}$ machine at stage 3 .

Constraints (4.11) defines the interval for the processing time on the machine $\mathrm{CC}_{k}$ at the stage 3 for a charge $c$.

Constraint (4.12) means that the sojourn time (transit) of a charge $c$ is limited.

Constraint (4.13) defines the interval of the inter-sequence setup times at stage 3 .

Constraint (4.14) defines the due dates for the charges that are processed on a CC device.

\subsection{The objective function}

First we formulate the total completion time on each $\mathrm{CC}_{k}\left(k=1, \ldots, m_{3}\right)$. Then we formulate the makespan $\left(C_{\max }\right)$ as the maximal total value of the completion time of the last sequence $S_{k}$ and the corresponding setup time for all the $\mathrm{CC}_{k}$ devices. This formulation allows us to minimize both the completion and setup times of the entire production process and by the way to reduce the waiting time between two successive sequences.

Hence the $C_{\max }$ has the following mathematical formulation:

$$
\mathrm{C}_{\max }:=\max _{1 \leq k \leq m_{3}}\left\{\sigma_{S_{k}}+x_{k, n_{S_{k}}}^{3}+p_{k, n_{S_{k}}}^{3}\right\}
$$

On one hand, the identification of charges is independent of the sequences. However, their order numbers in the corresponding sequence are preserved. On the other hand, for each device, the sequencing is achieved by mean of the charges assignment variables. Also, constraints (4.9), (4.12) and (4.14) ensure that the continuity at the last stage, the allowed sojourn (transit) times and the due dates are met. 
To solve the problem, we set the objective as to minimize the makespan $C_{\max }$ subject to all the identified constraints. The problem can formally be written as follows:

$\left(\min C_{\max }\right) \begin{cases}\mathrm{C}_{\max }^{\star}= & \text { Minimize } \mathrm{C}_{\max } \\ \text { Subject to } & (1)-(14) ; \\ & C_{\max } \geq \sigma_{S_{k}}+x_{k, n_{S_{k}}}^{3}+p_{k, n_{S_{k}}}^{3}, \forall k=1, \ldots, m_{3} ; \\ & x_{k, n_{S_{k}}}^{3}, p_{k, n_{S_{k}}}^{3}, \mathrm{C}_{\max } \geq 0, \sigma_{S_{k}} \in\left[\sigma_{s_{k}}^{\min }, \sigma_{s_{k}}^{\max }\right] .\end{cases}$

The MILP above brings a novelty by considering the makespan as the sum of the inter-sequence setup time at the last stage and the total completion time. This formulation permits: (i) to minimize the setup time values, (ii) to respect the continuity constraints and (iii) to tighten the charges flows in order to optimize the productivity.

The MILP can be extended to a multi-stage batch production with continuity constraints and setup times at the last stage. This latter is generalizing in turn the multi-stage hybrid flowshop with continuity constraints. The production is processed on multi-sequential production stages where each stage is guaranteeing a multicapacity of parallel machines. However, all the devices located on the first $(r-1)$ stages are allowed to process jobs of any sequence where at the last stage, the sequence jobs are processed on their dedicated machine. Continuity constraints are imposed at the last stage with new introduced decision variables that are the times setup between two successive sequences of a batch production. This constitutes a novelty in our MILP.

\section{A genetic Algorithm For the SCC With inter-Sequence DEPENDENT SETUP TIME}

For HFS problems, [30] were the first to apply a hybrid GA to the HFS with setup times. The method has been applied to minimize the makespan for a $m$-stages system with unrelated parallel machines, setup times and machines eligibility. For HFS problems, most of the GA approaches usually separate assignment and sequencing (see [29, 32]). Generally, these methods, are first sequencing by permutation flowshop procedures and then are assigning charges to devices at each stage by some priority rules or by machine availability. However, these strategies yield poor solutions quality in general. In the next sections, we detail the RGA based heuristic to solve the proposed problem.

\subsection{Encoding chromosomes and an initial solution}

To obtain high quality solutions, we used an encoding strategy which integrates both sequencing and assignment of the charges at stages 1 and 2 and continuity constraints at stage 3 . The chromosome is defined as a sequence of the total $N$ charges that are assigned to their CC machines. Each gene is represented by a couple $(k, c)$ meaning that a charge $c$ of a certain sequence is assigned to $\mathrm{CC}_{k}$. The gene has a machine assignment index $k$ that refers to $\mathrm{CC}_{k}$ and a sequencing index $c$ that refers to the charge of a certain sequence $s_{k}$.

So that $(1, c), c=1, \ldots, n_{1}$, represents all the genes associated with sequences to process on machine $\mathrm{CC}_{1}$, $(2, c)$ the sequences to be processed on machine $\mathrm{CC}_{2}, \ldots$ The total number of genes is the total number of charges $N=\sum_{k=1}^{m_{3}} n_{k}$ where $n_{k}=\sum_{s_{k}=1}^{S_{k}} n_{s_{k}}$.

At stage 1, the order in which the charges start their processing time is obtained by the position of the genes in the chromosomes, that means we assign charges to converters with regards to their availability and possible slowdown dates.

The chromosome shown in Figure 4 means that a total of 10 charges have to be processed on 3 CC machines $\left(\mathrm{CC}_{1}, \mathrm{CC}_{2}, \mathrm{CC}_{3}\right)$ such that $\mathrm{CC}_{1}$ must process a sequence of 4 charges, $\mathrm{CC}_{2}$ a sequence of 3 charges and $\mathrm{CC}_{3}$ has to process a sequence of 3 charges. For example, the couple $(3,1)$ represents the gene $(k, c)$ such that the machine $\mathrm{CC}_{(k=3)}$ has to process the first charge $c=1$. 


\begin{tabular}{|l|l|l|l|l|l|l|l|l|l|}
\hline$(1,1)$ & $(3,1)$ & $(2,1)$ & $(2,2)$ & $(1,2)$ & $(1,3)$ & $(3,2)$ & $(1,4)$ & $(3,3)$ & $(2,3)$ \\
\hline
\end{tabular}

Figure 4. A chromosome encoding configuration.

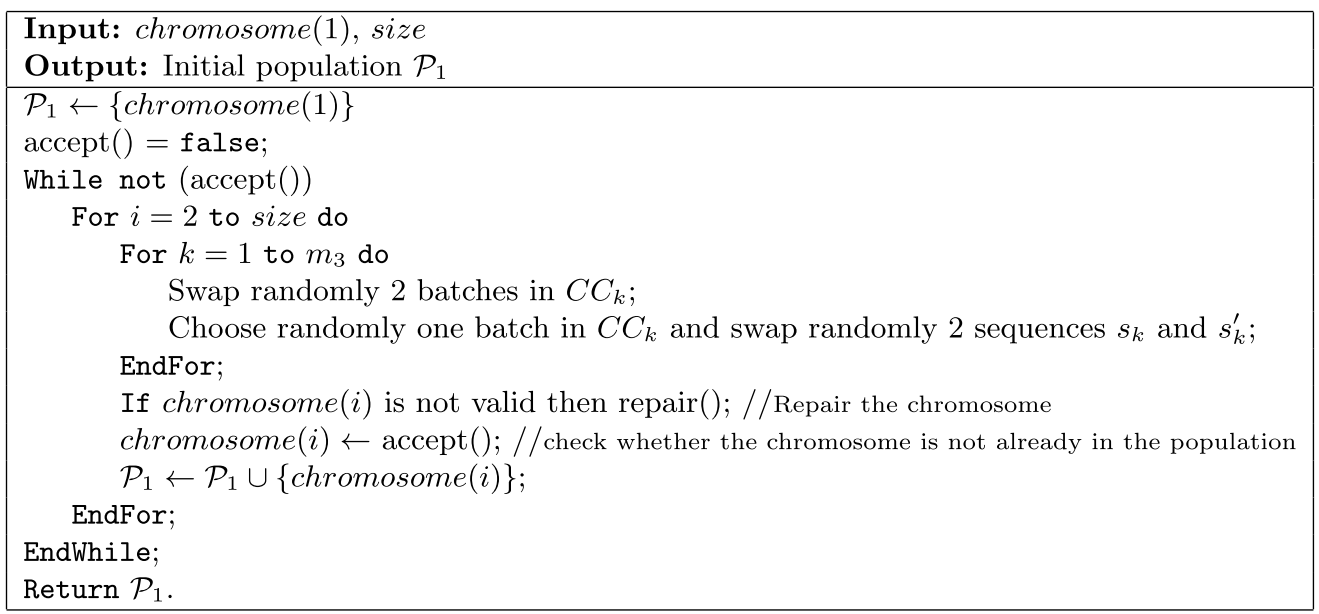

FiguRE 5. Gener(): an initial population $\mathcal{P}_{1}$.

In the following, we show how to generate a first feasible solution. We also consider the fitness function $f=\frac{1}{1+C_{\max }}$ to build fittest solutions. A chromosome is said to be healthier if it has the lowest makespan value $C_{\max }$. We add 1 to the denominator of the ratio to avoid a possible dividing by 0 . The schedule with the minimal makespan value is considered as the best solution.

We recall that for HFS it is not always easy to determine the start time at the first stage. Based on the assignment constraints (4.3) and (4.4) on one hand, and on the precedence constraints (4.6), (4.7) and (4.8) on the other hand, we compute (constraint (4.5)) the start time of the charges at the first stage according to the minimal sojourn time.

One can also use a heuristic procedure to fix the machine assignments and precedence relationships as parameters so that the obtained model carries only continuous variables that is easy to solve in this case. We call the first feasible solution obtained in Section 5.1 chromosome(1). Then from chromosome(1), a first population $\mathcal{P}_{1}$ is generated using the following steps:

Once a first feasible solution is obtained, we apply the Gener() procedure to generate an initial population $\mathcal{P}_{1}$ of size size (Fig. 5).

For each generated individual, we consider the objective function such that:

- to minimize the makespan $C_{\max }$,

- to meet with the continuity constraints at the CC stage,

- to reduce the charges sojourn times,

- to meet with due dates.

\subsection{GA operators}

\subsubsection{Crossover}

Several crossover techniques were developed by the past for GA. To ensure constructing feasible sequences while performing crossover operations, we use a tailored order crossover that is adapted from the classical OX operator. Our crossover operator consists: (i) to generate two children by swapping genes information of two 


\begin{tabular}{|c|c|c|c|c|c|c|c|c|c|}
\hline$(1,1)$ & $(3,1)$ & $(2,1)$ & $(1,2)$ & $(1,3)$ & $(3,2)$ & $(2,2)$ & $(1,4)$ & $(3,3)$ & $(1,5)$ \\
\hline \multicolumn{10}{|c|}{ parent 2} \\
\hline$(3,1)$ & $(2,1)$ & $(2,2)$ & $(1,1)$ & $(1,2)$ & $(3,2)$ & $(1,3)$ & $(1,4)$ & $(1,5)$ & $(3,3)$ \\
\hline \multicolumn{6}{|c|}{ portion 1} & \multicolumn{4}{|l|}{ cut } \\
\hline$(1,1)$ & $(3,1)$ & $(2,1)$ & $(1,2)$ & $(1,3)$ & $(3,2)$ & & & & \\
\hline \multicolumn{3}{|c|}{ portion 2} & & & & \multicolumn{4}{|c|}{ cut } \\
\hline$(3,1)$ & $(2,1)$ & $(2,2)$ & $(1,1)$ & $(1,2)$ & $(3,2)$ & & & & \\
\hline \multicolumn{10}{|c|}{ clean up 1} \\
\hline$(1,1)$ & $(3,1)$ & $(2,1)$ & $(1,2)$ & $(1,3)$ & $(3,2)$ & $(2,2)$ & $(1,4)$ & $(3,3)$ & $(1,5)$ \\
\hline
\end{tabular}

\begin{tabular}{l} 
clean up 2 \\
\begin{tabular}{|l|l|l|l|l|l|l|l|l|l|}
\hline$(3,1)$ & $(2,1)$ & $(2,2)$ & $(1,1)$ & $(1,2)$ & $(3,2)$ & $(1,3)$ & $(1,4)$ & $(1,5)$ & $(3,3)$ \\
\hline
\end{tabular} \\
survival 1 \\
\hline
\end{tabular}

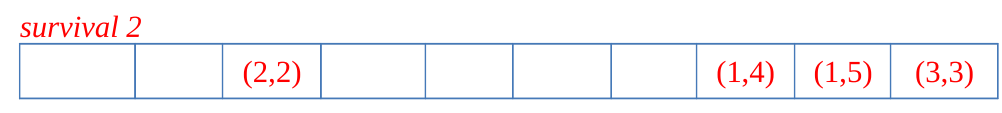

offspring 1
\begin{tabular}{|c|c|c|c|c|c|c|c|c|c|}
\hline$(1,1)$ & $(3,1)$ & $(2,1)$ & $(1,2)$ & $(1,3)$ & $(3,2)$ & $(2,2)$ & $(1,4)$ & $(1,5)$ & $(3,3)$ \\
\hline
\end{tabular}

offspring 2
\begin{tabular}{|c|c|c|c|c|c|c|c|c|c|}
\hline$(3,1)$ & $(2,1)$ & $(2,2)$ & $(1,1)$ & $(1,2)$ & $(3,2)$ & $(1,3)$ & $(1,4)$ & $(3,3)$ & $(1,5)$ \\
\hline
\end{tabular}

Figure 6. A crossover mechanism.

parents that are selected with a selection probability, (ii) to identify by mean of a random cut the left and right cut sides of each parent and (iii) to generate the children by selecting the first part of the first parent while keeping the order of the genes of the second parent.

The example (Fig. 6) shows how we adapt the crossover operation. We consider 2 chromosomes representing a configuration of 10 charges dedicated to $3 \mathrm{CC}$ where $\mathrm{CC}_{1}$ has to process 5 charges, $\mathrm{CC}_{2}$ has to process 2 charges and $\mathrm{CC}_{3}$ has to process 3 charges. The cut is selected at the 6 th gene.

The crossover operator works as follows:

- we randomly select a cut position;

- for each portion and starting from the left side, we remove one by one all the genes that are common with the other parent;

- we complete each survival with the remaining genes of the other survival in their recorded order to obtain an offspring.

We can see that the crossover is automatically ensuring the feasibility of the obtained chromosome since it is preserving the $\mathrm{CC}$ machines assignment at the last stage. 


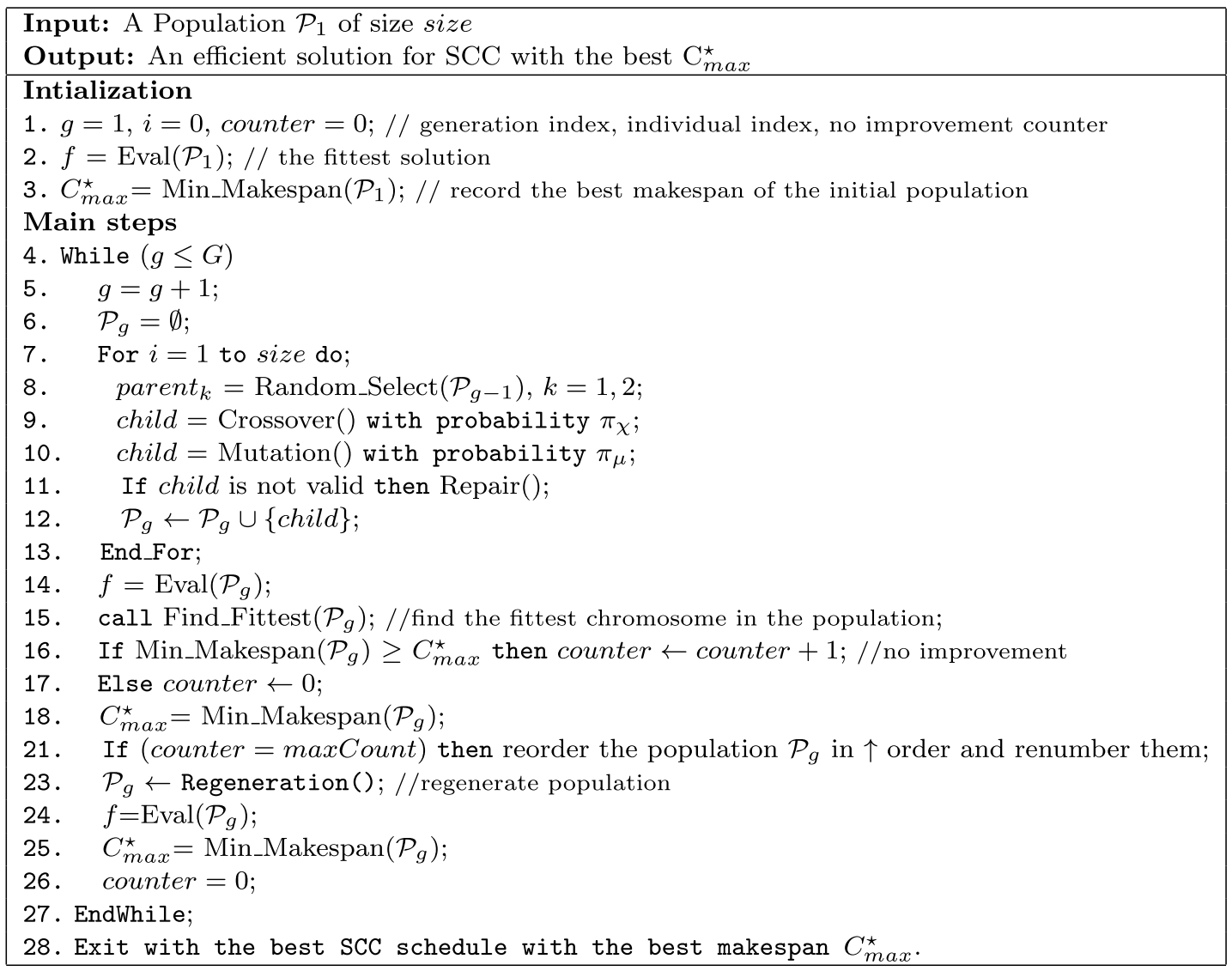

Figure 7. A RGA for SCC.

\subsubsection{Mutation}

We use some classical mutation operators (the exchange, swap or shift operators). We compute the mutation probability for each gene with regards to its position in the chromosome. Then, the mutation operator shifts a random selected position in the sequence and relocates it at a random position (at the left or the right) where the charges between the two shifted positions change also their positions regarding the shifted charges. If the obtained child is not valid, then a repairing step is performed.

\subsection{The GA algorithm}

We use the following parameters: the population size (size), the number of generations $(G)$, the selection probability, the crossover probability $\left(\pi_{\chi}\right)$ and the mutation probability $\left(\pi_{\mu}\right)$. The population evolves for a fixed number of generations. However we may encounter the difficulty such that for a given generation, the solution is not improved enough due to poor population diversity. In this case, we integrate a regeneration mechanism based on the idea proposed by [30].

A new feature for our RGA is that it is generalizing the regeneration mechanism since we adapt it to a set of jobs sequences. Also, at each generation, we record the value that realizes the minimum makespan $C_{\max }$ value. If for a certain number of iterations (or treshold) no improvement is observed for the fitness value, then we apply the regeneration mechanism to regenerate the population in order to escape local optimum (Fig. 7). 


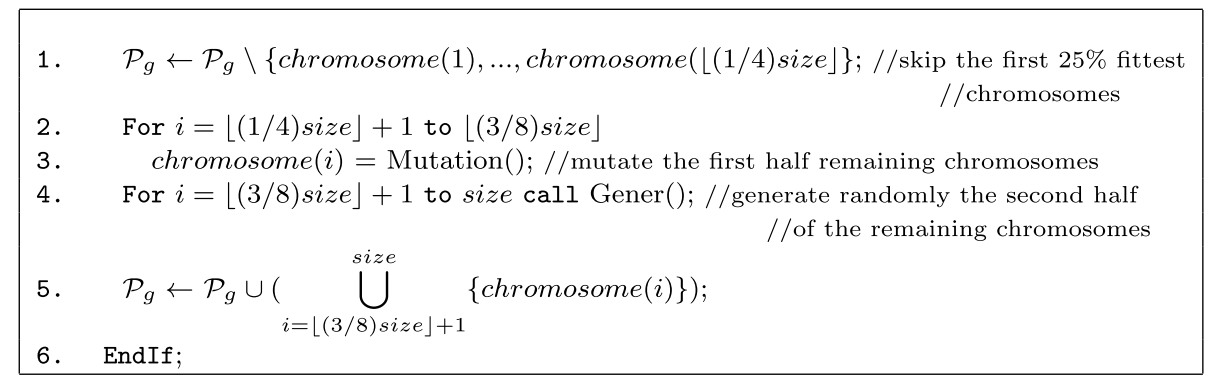

Figure 8. The Regeneration() procedure.

The $\operatorname{Eval}()$ function computes the fitness value of the individuals. Then GA generates at each generation $g$ a new population $\mathcal{P}_{g}$ by mating two random selected parents parent ${ }_{1}$ and parent $_{2}$ from $\mathcal{P}_{g-1}$. The selection is performed thanks to the selection probability $\frac{f_{i}}{\sum_{i=1}^{s i z e} f_{i}}$.

The derived GA namely RGA comprises 2 phases and is detailed as follows:

In the GA phase, we apply the Crossover() operator to generate a child noted child. Then, if the mutation probability $\pi_{\mu}$ is acceptable, we apply the Mutation() operator on child and we add child to $\mathcal{P}_{g}$.

The repairing is applied to eliminate infeasibility such as discontinuities and large sojourn times by modifying the sequences start time. We reiterate the process until we generate size chromosomes to add to the new population $\mathcal{P}_{g}$. We evaluate the individuals and select the fittest one as the solution (best $C_{\max }$ ) to SCC.

Another new feature of our approach resides in the procedure Find_Fittest() which uses the MILP model of Section 4.2 as a neighborhood search optimization to improve locally the solution. If the continuity and sojourn time constraints are not violated, then we reach a local optimum for the makespan and the SCC obtains an efficient solution. Otherwise, there exists no feasible solution in the considered neighborhood space.

At a given generation $g$ which is not improved enough, a counter of no improvement records the current state and is incremented by one unit until it reaches a maximum value fixed in advance, then the Regeneration() phase (Fig. 8) of the algorithm is activated (Steps 23-26).

\section{EXPERIMENTAL TESTS}

In this section, we detail the numerical experiments carried out in order to test the proposed approach. We have coded the GA with $\mathrm{C}++$ language on a Ubuntu Linux 14.04 Hp ProBook Core i3, 2.4 GHz and 6 Go of RAM. The CPU time, the completion time and the makespan are computed in seconds while the model run time is related to the instance configuration (sequences and charges numbers). Thus attempting to solve the SCC to its optimality may leads the computation time to grow fast with the size of the problem. Even though only small problems can be solved by mathematical programming techniques, there are still benefits to study the model in order to develop efficient approximate approaches.

The mathematical model that we present helps to better understand the problem structure and its complexity. However to the best of our knowledge, the model herein presented has not been studied so far. Consequently, no existing results are available in the literature to compare with our numerical results. We have based the design of our benchmark on the paper by [31] and other instances of large size were randomly generated with the same industrial context.

The population size size defines the number of chromosomes which conformed the population. If we have too small population sizes, the algorithm converges faster to a local optimum. In the case of a large population size, the computation time becomes disadvantageous. The number of generated populations $G$ stands for the number of evolutions steps to apply and a small number of generations usually leads to an early optimization break. Also a large number of generations is too time consuming and no possible improvement can be performed after a certain number of generations. 
TABLE 1. Cplex results for a $2 \mathrm{CC}$ problem with different sequences size.

\begin{tabular}{|c|c|c|c|c|}
\hline \multicolumn{2}{|c|}{ \# Inst. $\left(n_{1_{1}}, \ldots, n_{S_{1}}\right) \mathrm{x}\left(n_{1_{2}}, \ldots, n_{S_{2}}\right)$} & \multirow{2}{*}{$\frac{\mathrm{CPU}}{27.67}$} & \multicolumn{2}{|c|}{$\mathrm{CC}_{1}$ compl. time $\mathrm{CC}_{2}$ compl. time } \\
\hline 1 & $(5,5) \times(5,5)$ & & 887.58 & 864.43 \\
\hline 2 & $(14,21) \times(5,5,5)$ & 917.25 & 1087.01 & 974.26 \\
\hline 3 & $(15,10,12) \times(5,5,5)$ & out of mem. err. & $\mathrm{N} / \mathrm{A}$ & $\mathrm{N} / \mathrm{A}$ \\
\hline 4 & $(10,13,17,8) \times(5,5,5,5,5)$ & out of mem. err. & $\mathrm{N} / \mathrm{A}$ & $\mathrm{N} / \mathrm{A}$ \\
\hline 5 & $(18,11,23,15,27) \times(5,5,5,5,5,5,5,5,5,5)$ & out of mem. err. & $\mathrm{N} / \mathrm{A}$ & $\mathrm{N} / \mathrm{A}$ \\
\hline
\end{tabular}

TABle 2. Cplex results for a $3 \mathrm{CC}$ problem with different sequences size.

\begin{tabular}{|c|c|c|c|c|c|}
\hline \multicolumn{2}{|c|}{$\begin{aligned} & \# \text { Inst. }\left(n_{1_{1}}, \ldots, n_{S_{1}}\right) \times\left(n_{1_{2}}, \ldots, n_{S_{2}}\right) \\
& \times\left(n_{1_{3}}, \ldots, n_{S_{3}}\right) \\
&\end{aligned}$} & $\mathrm{CPU}$ & \multicolumn{3}{|c|}{$\mathrm{C}_{1}$ compl. time $\mathrm{C}_{2}$ compl. time $\mathrm{C}_{3}$ compl. time } \\
\hline 1 & $(5) \times(5) \times(5)$ & 2.56 & 363.45 & 396.78 & 382.88 \\
\hline 2 & $\begin{array}{l}(10,10) \times(10,10) \\
\quad \times(10,10)\end{array}$ & 1261.27 & 676.31 & 692.26 & 713.10 \\
\hline 3 & $\begin{array}{l}(5,15,19) \times(7,10,8) \\
\quad \times(5,7,12)\end{array}$ & 2321.38 & 1496.34 & 1389.38 & 1214.19 \\
\hline 4 & $\begin{array}{l}(5,5,8) \times(6,4) \\
\quad \times(5,4,5) \times(6,6,7)\end{array}$ & out of mem. err. & $\mathrm{N} / \mathrm{A}$ & $\mathrm{N} / \mathrm{A}$ & $\mathrm{N} / \mathrm{A}$ \\
\hline 5 & $\begin{array}{l}(5,15,5,10) \\
\quad \times(10,10,10,5) \times(5,5,5,5)\end{array}$ & out of mem. err. & $\mathrm{N} / \mathrm{A}$ & $\mathrm{N} / \mathrm{A}$ & $\mathrm{N} / \mathrm{A}$ \\
\hline 6 & $\begin{array}{l}(5,10,5,10,5) \\
\quad \times(10,10,10,5,5) \times(5,5,5,5,5)\end{array}$ & out of mem. err. & $\mathrm{N} / \mathrm{A}$ & $\mathrm{N} / \mathrm{A}$ & $\mathrm{N} / \mathrm{A}$ \\
\hline
\end{tabular}

TABLE 3. Optimization performance study for RGA and standard GA.

\begin{tabular}{|c|c|c|c|c|c|c|}
\hline Configuration & Pop. size & Approach & Av. $C_{\max }$ & Av. Std. Dev & Av. 100 & $\left(\frac{C_{\max _{\mathrm{RGA}}}-C_{\max _{\mathrm{GA}}}}{C_{\max _{\mathrm{GA}}}}\right)$ \\
\hline \multirow{2}{*}{$\begin{array}{c}2 \mathrm{CV}-2 \mathrm{RS}-2 \mathrm{CC} \\
(14,21) \times(5,5,5)\end{array}$} & 100 & $\begin{array}{l}\text { RGA } \\
\text { GA }\end{array}$ & $\begin{array}{l}1245.15 \\
1512.23\end{array}$ & 0.58 & \multicolumn{2}{|l|}{$-17.6 \%$} \\
\hline & 200 & $\begin{array}{l}\text { RGA } \\
\text { GA }\end{array}$ & $\begin{array}{l}1207.23 \\
1431.40\end{array}$ & $\begin{array}{l}0.59 \\
0.44\end{array}$ & \multicolumn{2}{|l|}{$-15.6 \%$} \\
\hline \multirow{2}{*}{$\begin{array}{c}\text { 3CV-3RS-3CC } \\
(15,15,19) \times(7,10,8)\end{array}$} & 100 & $\begin{array}{l}\text { RGA } \\
\text { GA }\end{array}$ & $\begin{array}{l}1625.54 \\
2125.17\end{array}$ & $\begin{array}{l}0.57 \\
0.46\end{array}$ & \multicolumn{2}{|l|}{$-23.5 \%$} \\
\hline & 200 & $\begin{array}{l}\text { RGA } \\
\text { GA }\end{array}$ & $\begin{array}{l}1572.71 \\
1932.35\end{array}$ & $\begin{array}{l}0.54 \\
0.38\end{array}$ & \multicolumn{2}{|l|}{$-18.6 \%$} \\
\hline
\end{tabular}

TABLE 4. Comparison of the averages consuming time for RGA and standard GA.

\begin{tabular}{llll}
\hline Configuration & Pop. size & Av. Comp. time for RGA & Av. Comp. time for std. GA \\
\hline 2CV-2RS-2CC & 100 & 1643.24 & 6812.27 \\
$(14,21) \times(5,5,5)$ & 200 & 1843.12 & 7207.13 \\
3CV-3RS-3CC $(15,15,19)$ & 100 & 1987.22 & 8145.30 \\
$\quad \times(7,10,8) \times(5,7,12)$ & 200 & 2317.38 & 9045.87 \\
\hline
\end{tabular}


TABle 5. A $C_{\max }$ sensitivity analysis for $\operatorname{SCC}([14,21] \times[5,5,5])$.

\begin{tabular}{llllll}
\hline Size & $C_{\max }^{\star}$ & $C_{\max }^{\star \star}$ & $\bar{C}_{\max }$ & sd & Err. \\
\hline 25 & 1230.202 & & 1231.642 & 0.737 & 2.670 \\
30 & 1223.023 & & 1224.186 & 1.002 & 2.071 \\
35 & 1219.332 & 1198.202 & 1219.922 & 0.442 & 1.763 \\
40 & 1213.063 & & 1214.439 & 1.217 & 1.240 \\
45 & 1200.001 & & 1201.783 & 0.770 & 0.150 \\
50 & 1198.202 & & 1199.277 & 0.933 & 0.0 \\
\hline
\end{tabular}

TABLE 6. A $C_{\max }$ sensitivity analysis for $\operatorname{SCC}([15,15,19] \times[7,10,8] \times[5,7,2])$.

\begin{tabular}{llllll}
\hline Size & $C_{\max }^{\star}$ & $C_{\max }^{\star \star}$ & $\bar{C}_{\max }$ & sd & Err. \\
\hline 25 & 1590.304 & & 1591.817 & 0.967 & 0.762 \\
30 & 1588.031 & & 1589.492 & 0.689 & 0.618 \\
35 & 1584.086 & & 1585.042 & 0.632 & 0.368 \\
40 & 1586.147 & 1578.274 & 1586,993 & 0.555 & 0.498 \\
45 & 1578.274 & & 1579.113 & 0.577 & 0.0 \\
50 & 1578.788 & & 1579.305 & 0.416 & 0.032 \\
\hline
\end{tabular}

The crossover probability $\pi_{\chi}$ stands for the intensity to pair chromosomes and the mutation probability $\pi_{\mu}$ determines the level to modify the configuration of the chromosomes. All these parameters needs to be well defined for high quality solutions.

As a first step of our study, we show the validity of the model and its hardness to obtain an optimal solution for medium and large size instances. We use a commercial solver (CPLEX V12.71) and we report the CPU time. All the tested instances by the solver are $2 \mathrm{CC}$ and $3 \mathrm{CC}$ configurations at the last stage (Tabs. 1 and 2).

The numerical results presented herein and based on a real-life sequences show the validity of our model for the planning of several sequences for a $2 \mathrm{CC}$ and a $3 \mathrm{CC}$ systems. CPLEx failed to solve some of the instances with $2 \mathrm{CC}$ and $3 \mathrm{CC}$ and for all the systems with $4 \mathrm{CC}$ and higher due to a lack of memory.

\subsection{Performance of the RGA}

To study the performance of the RGA, we have first applied the standard GA without Regeneration() procedure and have compared the obtained results to those of the RGA. We have run two tests with population sizes of 100 and 200 each (for a crossover probability $\pi_{\chi}=0.8$ and a mutation probability $\pi_{\mu}=0.03$ ) on a 2CV-2RS-2CV and a 3CV-3RS-3CC configurations. For each case, we used 10 sets of instances that have been tested 10 times repeatedly. In Table 3, we report the performance of RGA based an a comparison study of the average results from each test case (population size). We can observe that our approach is able to provide on average a smaller makespan $C_{\max }$ than the standard GA for the 2 population sizes and a lower standard deviation. The gaps between GA and RGA represent $-17.6 \%$ and $-15.6 \%$ for the $2 \mathrm{CV}-2 \mathrm{RS}-2 \mathrm{CC}$ and $-23.5 \%$ and $-18.6 \%$ for the $3 \mathrm{CV}-3 \mathrm{RS}-3 \mathrm{CC}$ configuration for 100 and 200 individuals respectively. From these outputs, we can easily state that the solutions obtained by RGA are likely to be of better quality that those obtained by the standard GA.

Moreover, we have considered the computational time to measure the performance of the RGA. We reported in Table 4 the average time obtained for all the tests cases. From these results, we can remark that the RGA is nearly 4 times less consuming time than the standard GA. We can explain it by the fact that our algorithm manages well the SCC scheduling by saving the time to build a schedule for a batch. 
TABle 7. A $C_{\max }$ sensitivity analysis for $\operatorname{SCC}([5,5,8] \times[6,4] \times[5,4,5] \times[6,6,7])$.

\begin{tabular}{llllll}
\hline Size & $C_{\max }^{\star}$ & $C_{\max }^{\star \star}$ & $\bar{C}_{\max }$ & $\mathrm{sd}$ & Err. \\
\hline 25 & 2611.066 & & 2611.406 & 0.246 & 0.717 \\
30 & 2604.069 & & 2605.442 & 0.888 & 0.447 \\
35 & 2601.176 & & 2602.449 & 0.938 & 0.336 \\
40 & 2594.216 & 2592.458 & 2595.521 & 0.927 & 0.067 \\
45 & 2593.058 & & 2594.143 & 0.732 & 0.023 \\
50 & 2592.458 & & 2593.048 & 0.400 & 0.0 \\
\hline
\end{tabular}

\subsection{Sensitivity analysis and parameters setting}

To test the efficiency of our algorithm, we make a sensitivity analysis study. The aim is:

- to test the influence of the population size;

- to investigate the relationship between the total number of populations for a population with a fixed size.

For each chosen instance, we have launched 20 runs. Tables 5-8 detail the best makespan noted $\mathrm{C}_{\max }^{\star}$ for each fixed size, the best found makespan noted $C_{\max }^{\star \star}$ which corresponds to the best settings for all the fixed sizes, the average makespan of the 20 runs noted $\bar{C}_{\max }$, the standard deviation noted sd and the relative percentage error noted err.

Since we do not know any optimal solution for the tested instances or any alternative approach that has been tested by the past, we compare the results with the best found solution so far. We compute the relative percentage error as err $=100 \times \frac{C_{\max }^{\star}-C_{\max }^{\star \star}}{C_{\max }^{\star}}$ and compute the standard deviation noted sd.

\subsubsection{Sensitivity analysis of the population size}

We have set the number of populations to $G=150$, varied the size of each population in the set $\{25,30,35,40,45,50\}$ and we have run the RGA algorithm 20 times. Tables $5-7$ give the results for the three instances $\operatorname{SCC}([14,21] \times[5,5,5]), \operatorname{SCC}([15,15,19] \times[7,10,8] \times[5,7,2])$ and $\operatorname{SCC}([5,5,8] \times[6,4] \times[5,4,5] \times$ $[6,6,7])$.

For the 3 problem instances, RGA has been running 20 times for each chosen population size (size). $C_{\max }^{\star}$ corresponds to the best found solution so far among the 20 computed ones and $C_{\text {max }}^{\star \star}$ corresponds to the best settings.

Note that as we increase the size of the population, the variance value $\left(\mathrm{sd}^{2}\right)$ decreases quickly to zero value from size $=30$ to size $=40$ and with a small decrease from size $=40$ to size $=50$. This means that from a certain size of the population RGA gives the solutions for 20 runs with comparable quality. For example, for the problem instance $\operatorname{SCC}([15,15,19] \times[7,10,8] \times[5,7,2])$, the algorithm always gives the best $C_{\max }^{\star}$ for sizes value starting from 150 . The size value can then be limited to 150 with no need to increase it more. This allows us to save computational time.

TABLE 8. A $C_{\max }$ sensitivity analysis for $\operatorname{SCC}([5,5,8] \times[6,4] \times[5,4,5] \times[6,6,7])$.

\begin{tabular}{lllllll}
\hline$G$ & $C_{\max }^{\star}$ & $C_{\max }^{\star \star}$ & $\bar{C}_{\max }$ & sd & Err. & CPU(s) \\
\hline 50 & 2609.10 & & 2699.23 & 1.27 & 4.25 & 863.8 \\
100 & 2663.02 & & 2665.61 & 2.28 & 2.93 & 1014.1 \\
150 & 2599.96 & 2587.04 & 2602.44 & 1.02 & 0.49 & 1523.5 \\
250 & 2590.25 & & 2592.28 & 3.32 & 0.12 & 1987.4 \\
300 & 2587.04 & & 2590.79 & 2.54 & 0.0 & 2021.5 \\
\hline
\end{tabular}




\subsubsection{Sensitivity analysis of the number of populations}

For this study, we set the population size to 50. Table 8 shows the best $C_{\max }^{\star}$ resulting from 20 runs of the RGA algorithm for a SCC instance and for different number of generations $G$ taken from the set $\{50,150,200,250,300\}$. The Computation time was also reported.

Table 8 shows that the RGA is able to obtain good solutions with an "acceptable" computational time. The best generations calibration is around 150. For big generations number, RGA consumes huge amount of time without a substantial improvement of the solution. Also for a small generations number, RGA leads "rapidily" to a solution whose quality is less than the best known so far.

\subsection{Numerical tests}

For the model presented in Section 4.2, we have developed and tested a 2-phases RGA based heuristic on a set of SCC real instances and other randomly generated ones that simulate a production plan. For memory amount considerations, we have limited our examples to 2 SCC configurations. The first configuration is a 2 CC machines and the second one is a 3 CC machines. All the related parameters to the 2 SCC systems are known and the solution is highlighted in bold characters.

TABLE 9. The RGA paramaters.

\begin{tabular}{ll}
\hline Parameters & RGA \\
\hline \# of generations $G$ & 150 \\
Population size size & 50 \\
Crossover prob. $\pi_{\chi}$ & 0.8 \\
Mutation prob. $\pi_{\mu}$ & 0.03 \\
Regeneration() maxCount & 25 \\
\hline
\end{tabular}

TABLE 10. Performance comparaison between RGA and HCGA.

\begin{tabular}{|c|c|c|c|c|c|c|}
\hline$N \times 3 \times m$ & HCGA & RGA & \%Improvement & $\mathrm{CPU}_{\mathrm{HCGA}}(\mathrm{s})$ & CPU RGA (s) & \%Improvement \\
\hline $60 \times 3 \times 4$ & 227.0140 & 227.0140 & 0.0000 & 27.5000 & 11.3792 & -0.5862 \\
\hline $60 \times 3 \times 8$ & 126.9580 & 126.9580 & 0.0000 & 40.1000 & 21.4347 & -0.4656 \\
\hline $60 \times 3 \times 12$ & 94.3647 & 94.0580 & -0.0033 & 65.5000 & 39.9107 & -0.3907 \\
\hline $60 \times 3 \times 16$ & 78.1323 & 77.7080 & -0.0054 & 95.4000 & 63.2675 & -0.3368 \\
\hline $80 \times 3 \times 4$ & 319.0330 & 319.0330 & 0.0000 & 36.4000 & 34.7705 & -0.0448 \\
\hline $80 \times 3 \times 8$ & 174.6420 & 174.6420 & 0.0000 & 65.3000 & 55.7230 & -0.1467 \\
\hline $80 \times 3 \times 12$ & 127.4397 & 127.0000 & -0.0035 & 92.8000 & 78.2667 & -0.1566 \\
\hline $80 \times 3 \times 16$ & 103.8413 & 103.3000 & -0.0052 & 122.6000 & 89.2809 & -0.2718 \\
\hline $80 \times 3 \times 20$ & 90.1456 & 89.4000 & -0.0083 & 238.8000 & 234.5156 & -0.0179 \\
\hline $100 \times 3 \times 8$ & 338.7683 & 338.5000 & -0.0008 & 79.3000 & 78.8036 & -0.0063 \\
\hline $100 \times 3 \times 12$ & 241.1030 & 240.0000 & -0.0046 & 155.8000 & 87.5064 & -0.4383 \\
\hline $100 \times 3 \times 16$ & 192.7576 & 191.8447 & -0.0047 & 247.6000 & 170.8258 & -0.3101 \\
\hline $100 \times 3 \times 20$ & 164.4252 & 163.7000 & -0.0044 & 498.8000 & 455.9293 & -0.0859 \\
\hline $120 \times 3 \times 6$ & 334.6000 & 329.9237 & -0.0140 & 698.0000 & 533.5807 & -0.2356 \\
\hline $120 \times 3 \times 10$ & 287.5000 & 283.9800 & -0.0122 & 765.0000 & 613.0737 & -0.1986 \\
\hline $120 \times 3 \times 14$ & 423.7000 & 420.6253 & -0.0073 & 987.0000 & 586.7541 & -0.4055 \\
\hline $150 \times 3 \times 4$ & 298.6000 & 294.7603 & -0.0129 & 1002.0000 & 591.6787 & -0.4095 \\
\hline $150 \times 3 \times 8$ & 409.7000 & 406.3901 & -0.0081 & 1579.0000 & 918.2093 & -0.4185 \\
\hline $150 \times 3 \times 10$ & 475.6700 & 469.9805 & -0.0120 & 1987.0000 & 1894.6503 & -0.0465 \\
\hline Average & & & -0.0056 & & & -0.2501 \\
\hline
\end{tabular}


TABLE 11. A RGA results for $\mathrm{CC}_{1}$ for Problem $\mathrm{SCC}([14,21] \times[5,5,5])$.

\begin{tabular}{|c|c|c|c|c|c|c|c|c|}
\hline Charge $(k, c)$ & $x_{k, c}^{1}$ & $\mathrm{CV}_{i}$ & $x_{k, c}^{2}$ & $x_{k, c}^{3}$ & $p_{k, c}^{3}$ & Comp. time & Sojourn & cont. \\
\hline 1 & 30 & 1 & 89 & 126 & 24 & 150 & 52 & $* *$ \\
\hline 2 & 50 & 1 & 112.3698 & 149.3698 & 42.9453 & 192.3151 & 55.3698 & $<0.1$ \\
\hline 3 & 94 & 2 & 154.6849 & 191.6849 & 42.9453 & 234.6302 & 53.6849 & $<0.1$ \\
\hline 4 & 138 & 1 & 197.0000 & 234.0000 & 30.3619 & 264.3619 & 52 & $<0.1$ \\
\hline 5 & 162 & 2 & 226.7317 & 263.7317 & 40.8049 & 304.5366 & 57.7317 & $<0.1$ \\
\hline 6 & 206 & 2 & 266.9064 & 303.9064 & 42.9453 & 346.8517 & 53.9064 & $<0.1$ \\
\hline 7 & 226 & 2 & 309.2215 & 346.2215 & 30.3619 & 376.5834 & 76.2215 & $<0.1$ \\
\hline 8 & 270 & 2 & 338.9532 & 375.9532 & 30.3619 & 406.3151 & 61.9532 & $<0.1$ \\
\hline 9 & 294 & 1 & 368.6849 & 405.6849 & 42.9453 & 448.6302 & 67.6849 & $<0.1$ \\
\hline 10 & 314 & 1 & 411 & 448 & 42.9453 & 490.9453 & 90 & $<0.1$ \\
\hline 11 & 382 & 2 & 453.3151 & 490.3151 & 28.5115 & 518.8266 & 64.3151 & $<0.1$ \\
\hline 12 & 402 & 1 & 475.3151 & 518.1964 & 28.5115 & 546.7079 & 72.1964 & $<0.1$ \\
\hline 13 & 426 & 1 & 509.0777 & 546.0777 & 35.8389 & 581.9166 & 76.0777 & $<0.1$ \\
\hline 14 & 470 & 2 & 531.0777 & 581.2864 & 37.3438 & 648.6302 & 67.2864 & $<0.1$ \\
\hline 1 & 514 & 2 & 573 & 648 & 20.6302 & 668.6302 & 90 & $* *$ \\
\hline 2 & 534 & 2 & 631 & 668 & 24.6302 & 692.6302 & 90 & $<0.1$ \\
\hline 3 & 558 & 1 & 655 & 692 & 36.6641 & 728.6641 & 90 & $<0.1$ \\
\hline 4 & 602 & 2 & 677 & 728.0339 & 23.8634 & 751.8973 & 82.0339 & $<0.1$ \\
\hline 5 & 646 & 1 & 705 & 751.2671 & 37.8265 & 789.0936 & 61.2671 & $<0.1$ \\
\hline 6 & 666 & 2 & 751.4634 & 788.4634 & 24.9705 & 813.4339 & 78.4634 & $<0.1$ \\
\hline 7 & 710 & 1 & 773.4634 & 812.8037 & 37.8265 & 850.6302 & 58.8037 & $<0.1$ \\
\hline 8 & 754 & 2 & 813 & 850 & 39.0664 & 889.0664 & 52 & $<0.1$ \\
\hline 9 & 778 & 1 & 837 & 888.4362 & 28.1953 & 916.6315 & 66.4362 & $<0.1$ \\
\hline 10 & 798 & 1 & 879.0013 & 916.0013 & 23.2397 & 939.241 & 74.0013 & $<0.1$ \\
\hline 11 & 842 & 2 & 901.6108 & 938.6108 & 24.0194 & 962.6302 & 52.6108 & $<0.1$ \\
\hline 12 & 866 & 2 & 925 & 962 & 24.0194 & 986.0194 & 52 & $<0.1$ \\
\hline 13 & 886 & 1 & 947 & 985.3892 & 24.0194 & 1009.4086 & 55.3892 & $<0.1$ \\
\hline 14 & 910 & 1 & 969 & 1008.7784 & 24.0194 & 1032.7978 & 54.7784 & $<0.1$ \\
\hline 15 & 930 & 1 & 991 & 1032.1676 & 24.0194 & 1056.187 & 58.1676 & $<0.1$ \\
\hline 16 & 954 & 2 & 1018.5568 & 1055.5568 & 24.0194 & 1079.5762 & 57.5568 & $<0.1$ \\
\hline 17 & 974 & 2 & 1041.946 & 1078.946 & 24.0194 & 1102.9654 & 60.946 & $<0.1$ \\
\hline 18 & 998 & 1 & 1063.946 & 1102.3352 & 24.0194 & 1126.3546 & 60.3352 & $<0.1$ \\
\hline 19 & 1018 & 1 & 1088.7244 & 1125.7244 & 27.1379 & 1152.8623 & 63.7244 & $<0.1$ \\
\hline 20 & 1042 & 2 & 1115.2321 & 1152.2321 & 36.7771 & 1189.0092 & 66.2321 & $<0.1$ \\
\hline 21 & 1062 & 1 & 1151.379 & 1188.379 & 36.7771 & 1225.1561 & 82.379 & $<0.1$ \\
\hline
\end{tabular}

The analysis study (Sect. 6.2) allows to better tune the parameters. Table 9 summarizes the parameters settings for RGA.

At a first stage and based on the above tuned parameters, we have tested the performance of our RGA on some HFS benchmark taken from [33] and another generated one.

We have adapted the RGA algorithm to the HFS model in order to solve a set of instances and their results are presented in Table 10.

As it was noted above, a SCC problem is a particular HFS problem with 3 stages. A HFS problem is such that jobs are single and are not belonging to a sequence. The batch is simply a set of jobs while in a SCC configuration, a batch is represented by a set of sequences of charges to process.

Table 10 presents the performance of RGA with regards to HCGA presented in [33]. To do so, we solve each combination of the total number of jobs in the system $(N=60,80,100,120$ and 150 and total number of 
TABLE 12. A RGA results for $\mathrm{CC}_{2}$ for $\mathrm{SCC}([14,21] \times[5,5,5])$.

\begin{tabular}{lllllllll}
\hline Charge $(k, c)$ & $x_{k, c}^{1}$ & $\mathrm{CV}_{i}$ & $x_{k, c}^{2}$ & $x_{k, c}^{3}$ & $p_{k, c}^{3} 3$ & Comp. time & Sojourn & cont. \\
\hline 1 & 15 & 2 & 74 & 121 & 41.5 & 162.5 & 62 & $* *$ \\
2 & 35 & 1 & 115.5976 & 162.5976 & 58.1391 & 220.7367 & 83.5976 & $<0.1$ \\
3 & 103 & 1 & 173.8343 & 220.8343 & 58.1391 & 278.9734 & 73.8343 & $<0.1$ \\
4 & 147 & 2 & 232.071 & 279.071 & 59.959 & 339.03 & 88.071 & $<0.1$ \\
5 & 211 & 1 & 292.1276 & 339.1276 & 59.959 & 399.0866 & 84.1276 & $<0.1$ \\
1 & 279 & 2 & 382.1842 & 429.1842 & 44.1837 & 503.3679 & 106.1842 & $* *$ \\
2 & 367 & 2 & 426 & 473.4655 & 61.8963 & 535.3618 & 62.4655 & $<0.1$ \\
3 & 387 & 1 & 458 & 535.4594 & 61.8963 & 597.3557 & 104.4594 & $<0.1$ \\
4 & 475 & 1 & 534 & 597.4533 & 61.8963 & 659.3496 & 78.4533 & $<0.1$ \\
5 & 519 & 1 & 578 & 659.4472 & 63.5079 & 722.9551 & 96.4472 & $<0.1$ \\
1 & 607 & 2 & 706.0527 & 753.0527 & 39.123 & 822.1757 & 102.0527 & $* *$ \\
2 & 651 & 2 & 738.0527 & 792.2733 & 54.8113 & 847.0846 & 97.2733 & $<0.1$ \\
3 & 719 & 2 & 800.1822 & 847.1822 & 54.8113 & 901.9935 & 84.1822 & $<0.1$ \\
4 & 763 & 1 & 855.0911 & 902.0911 & 54.8113 & 956.9024 & 95.0911 & $<0.1$ \\
5 & 851 & 2 & 910 & 957 & 54.8113 & 1011.8113 & 62 & $<0.1$ \\
\hline
\end{tabular}

machines $m=m_{1}+m_{2}+m_{3}=4,8,12,16$ and 20) with no specification on the number of machines $m_{r}$ per stage $r=1, \ldots, 3$.

As we have noted above, a SCC problem is a HFS (FFS) problem where jobs are single and are not belonging to a sequence. The batch is simply a set of jobs where in a SCC configuration, a batch is a set of sequences of charges to process.

Table 10 presents the performance of RGA with regards to HCGA presented in [33]. To do so, we solve each combination of number of jobs $n=60,80,100,120$ and 150 and number of machines $m=4,8,12,16$ and 20. We report the solution obtained by both CGHA and RGA in columns 2 and 3. Column 4 represents the improvement rate recorded by RGA with regards to HCGA. The average CPU times consumed by both HCGA and RGA are shown in columns 6 and 7 .

We report the solutions obtained by both CGHA and RGA in columns 2 and 3. Column 4 represents the improvement rate recorded by RGA with regards to HCGA. The average CPU times consumed by both HCGA and RGA are shown in columns 5 and 6.

For medium instances, RGA obtain on average the same solution found by CGHA but in a very small time while for large size instances RGA performs better and in a small time as well. The improvement average for the solution is about $5.6 \%$ (column 4) and the improvement average for the CPU consuming time is about $25 \%$ (column 7) which shows that RGA is much more faster than HCGA.

For each Tables (11 and 15), column 1 represents the charge as a couple representing the dedicated CC and the charge number of a sequence, columns 2-4-5 represent the computed start times $x_{k, c}^{r}(r=1, \ldots, 3)$ at each stage $\left(\mathrm{CV}_{i}-\mathrm{RS}_{j}-\mathrm{CC}_{k}\right)$ of a charge. Column 3 shows the $\mathrm{CV}_{i}$ number for which a charge is assigned at the first stage. Column 6 represents the computed processing time $p_{k, c}^{3}$ of a charge at the last stage $\mathrm{CC}_{k}$. Columns 7-8-9 represent respectively the completion time, the sojourn (transit) time and the continuity constraints for each charge $c$ dedicated to $\mathrm{CC}_{k}$.

We have run our numerical tests in order to study the behavior of the GA algorithm when its is running on problem instances of several types and configurations.

From Tables 11 and 12, the maximum completion times for both CC devices are respectively equal to 1225.15 and 1011.81. Thus the makespan $C_{\max }$ is equal to $1225.15 \mathrm{~min}$ of the production order. This efficient solution is reached for the charge number 21 of the sequence number 2 on $\mathrm{CC}_{1}$ device. Our approach was able to compute the obtained solution with a CPU time of $2321.60 \mathrm{~s}$. 
TABle 13. A RGA results for $\mathrm{CC}_{1}$ for $\operatorname{SCC}([15,15,19] \times[7,10,8] \times[5,7,12])$.

\begin{tabular}{|c|c|c|c|c|c|c|c|c|}
\hline Charge $(k, c)$ & $x_{k, c}^{1}$ & $\mathrm{CV}_{i}$ & $x_{k, c}^{2}$ & $x_{k, c}^{3}$ & $p_{k, c}^{3} 3$ & Comp. time & Sojourn & Cont. \\
\hline 1 & 10 & 1 & 69 & 106 & 21.3 & 127.3 & 52 & $* *$ \\
\hline 2 & 24 & 3 & 89 & 127.24 & 41.094 & 168.334 & 59.24 & $<0.1$ \\
\hline 3 & 68 & 1 & 127 & 168.27 & 29.795 & 198.065 & 56.27 & $<0.1$ \\
\hline 4 & 104 & 2 & 163 & 198 & 37.385 & 235.385 & 50 & $<0.1$ \\
\hline 5 & 112 & 3 & 200.32 & 235.32 & 29.795 & 265.115 & 79.32 & $<0.1$ \\
\hline 6 & 148 & 2 & 225 & 265.05 & 29.795 & 294.845 & 73.05 & $<0.1$ \\
\hline 7 & 186 & 2 & 245 & 294.79 & 29.795 & 324.585 & 64.79 & $<0.1$ \\
\hline 8 & 200 & 1 & 289.52 & 324.52 & 29.795 & 354.315 & 80.52 & $<0.1$ \\
\hline 9 & 244 & 3 & 309.52 & 354.25 & 27.944 & 382.194 & 66.25 & $<0.1$ \\
\hline 10 & 274 & 3 & 333 & 382.13 & 27.944 & 410.074 & 64.13 & $<0.1$ \\
\hline 11 & 288 & 1 & 353 & 410.01 & 27.944 & 437.954 & 78.01 & $<0.1$ \\
\hline 12 & 324 & 3 & 402.89 & 437.89 & 27.944 & 465.834 & 69.89 & $<0.1$ \\
\hline 13 & 332 & 2 & 430.77 & 465.77 & 35.272 & 501.042 & 89.77 & $<0.1$ \\
\hline 14 & 368 & 1 & 465.98 & 500.98 & 35.272 & 536.252 & 88.98 & $<0.1$ \\
\hline 15 & 412 & 2 & 485.98 & 536.19 & 16.647 & 602.837 & 80.19 & $<0.1$ \\
\hline 1 & 450 & 3 & 567.78 & 602.78 & 22.556 & 625.336 & 108.78 & $* *$ \\
\hline 2 & 464 & 1 & 590.27 & 625.27 & 22.556 & 647.826 & 117.27 & $<0.1$ \\
\hline 3 & 500 & 2 & 612.76 & 647.76 & 23.296 & 671.056 & 103.76 & $<0.1$ \\
\hline 4 & 508 & 1 & 636 & 671 & 22.565 & 693.565 & 119 & $<0.1$ \\
\hline 5 & 544 & 3 & 658.5 & 693.5 & 22.565 & 716.065 & 105.5 & $<0.1$ \\
\hline 6 & 552 & 1 & 681 & 716 & 23.527 & 739.527 & 120 & $<0.1$ \\
\hline 7 & 582 & 2 & 701 & 739.46 & 22.672 & 762.132 & 113.46 & $<0.1$ \\
\hline 8 & 632 & 3 & 721 & 762.07 & 22.672 & 784.742 & 86.07 & $<0.1$ \\
\hline 9 & 670 & 1 & 749.68 & 784.68 & 22.672 & 807.352 & 70.68 & $<0.1$ \\
\hline 10 & 684 & 2 & 772.29 & 807.29 & 23.452 & 830.742 & 79.29 & $<0.1$ \\
\hline 11 & 714 & 2 & 795.68 & 830.68 & 23.452 & 854.132 & 72.68 & $<0.1$ \\
\hline 12 & 758 & 1 & 819.07 & 854.07 & 23.452 & 877.522 & 52.07 & $<0.1$ \\
\hline 13 & 772 & 1 & 842.46 & 877.46 & 39.825 & 917.285 & 61.46 & $<0.1$ \\
\hline 14 & 808 & 3 & 882.22 & 917.22 & 23.452 & 940.672 & 65.22 & $<0.1$ \\
\hline 15 & 816 & 1 & 905.61 & 940.61 & 23.452 & 1014.062 & 80.61 & $<0.1$ \\
\hline 1 & 904 & 3 & 979 & 1014 & 25.474 & 1039.474 & 66 & $* *$ \\
\hline 2 & 940 & 2 & 999 & 1039.4 & 23.452 & 1062.852 & 55.4 & $<0.1$ \\
\hline 3 & 948 & 3 & 1027.8 & 1062.8 & 39.825 & 1102.625 & 70.8 & $<0.1$ \\
\hline 4 & 992 & 1 & 1067.6 & 1102.6 & 39.825 & 1142.425 & 66.6 & $<0.1$ \\
\hline 5 & 1028 & 3 & 1107.3 & 1142.3 & 39.825 & 1182.125 & 70.3 & $<0.1$ \\
\hline 6 & 1080 & 2 & 1147.1 & 1182.1 & 23.452 & 1205.552 & 58.1 & $<0.1$ \\
\hline 7 & 1110 & 3 & 1169 & 1205.5 & 23.452 & 1228.952 & 51.5 & $<0.1$ \\
\hline 8 & 1124 & 1 & 1193.9 & 1228.9 & 23.452 & 1252.352 & 60.9 & $<0.1$ \\
\hline 9 & 1154 & 2 & 1217.3 & 1252.3 & 23.452 & 1275.752 & 54.3 & $<0.1$ \\
\hline 10 & 1168 & 1 & 1240.6 & 1275.6 & 26.571 & 1302.171 & 63.6 & $<0.1$ \\
\hline 11 & 1198 & 2 & 1267.1 & 1302.1 & 36.21 & 1338.31 & 60.1 & $<0.1$ \\
\hline 12 & 1212 & 1 & 1303.3 & 1338.3 & 36.21 & 1374.51 & 82.3 & $<0.1$ \\
\hline 13 & 1242 & 3 & 1339.4 & 1374.4 & 23.452 & 1397.852 & 88.4 & $<0.1$ \\
\hline 14 & 1292 & 2 & 1362.8 & 1397.8 & 23.452 & 1421.252 & 61.8 & $<0.1$ \\
\hline 15 & 1300 & 3 & 1386.2 & 1421.2 & 23.452 & 1444.652 & 77.2 & $<0.1$ \\
\hline 16 & 1344 & 2 & 1409.6 & 1444.6 & 23.452 & 1468.052 & 56.6 & $<0.1$ \\
\hline 17 & 1374 & 3 & 1433 & 1468 & 26.571 & 1494.571 & 50 & $<0.1$ \\
\hline 18 & 1388 & 2 & 1453 & 1494.5 & 36.21 & 1530.71 & 62.5 & $<0.1$ \\
\hline 19 & 1424 & 3 & 1495.7 & 1530.7 & 36.21 & 1566.91 & 62.7 & $<0.1$ \\
\hline
\end{tabular}


TABle 14. A RGA results for $\mathrm{CC}_{2}$ for $\operatorname{SCC}([15,15,19] \times[7,10,8] \times[5,7,12])$.

\begin{tabular}{lllllllll}
\hline Charge $(k, c)$ & $x_{k, c}^{1}$ & $\mathrm{CV}_{i}$ & $x_{k, c}^{2}$ & $x_{k, c}^{3}$ & $p_{k, c}^{3} 3$ & Comp. time & Sojourn & Cont. \\
\hline 1 & 25 & 1 & 84 & 124 & 43.85 & 167.85 & 54 & $* *$ \\
2 & 69 & 2 & 128 & 168 & 69.734 & 237.734 & 54 & $<0.1$ \\
3 & 127 & 3 & 186 & 237.88 & 61.966 & 299.846 & 65.88 & $<0.1$ \\
4 & 201 & 2 & 260 & 300 & 59.907 & 359.907 & 54 & $<0.1$ \\
5 & 251 & 3 & 320.06 & 360.06 & 71.918 & 431.978 & 64.06 & $<0.1$ \\
6 & 333 & 1 & 392.12 & 432.12 & 46.94 & 479.06 & 54.12 & $<0.1$ \\
7 & 377 & 3 & 439.21 & 479.21 & 74.243 & 583.453 & 57.21 & $<0.1$ \\
1 & 427 & 1 & 543.61 & 583.61 & 74.243 & 657.853 & 111.61 & $* *$ \\
2 & 559 & 1 & 618 & 658 & 61.844 & 719.844 & 54 & $<0.1$ \\
3 & 611 & 2 & 679.99 & 719.99 & 61.844 & 781.834 & 63.99 & $<0.1$ \\
4 & 647 & 2 & 741.99 & 781.99 & 39.071 & 821.061 & 89.99 & $<0.1$ \\
5 & 699 & 1 & 781.21 & 821.21 & 54.759 & 875.969 & 77.21 & $<0.1$ \\
6 & 735 & 3 & 836.12 & 876.12 & 54.759 & 930.879 & 96.12 & $<0.1$ \\
7 & 817 & 2 & 891.03 & 931.03 & 54.759 & 985.789 & 69.03 & $<0.1$ \\
8 & 867 & 1 & 945.94 & 985.9 & 54.759 & 1040.659 & 73.9 & $<0.1$ \\
9 & 905 & 3 & 1000.8 & 1040.8 & 39.071 & 1079.871 & 90.8 & $<0.1$ \\
10 & 949 & 2 & 1040.1 & 1080.1 & 54.759 & 1164.859 & 86.1 & $<0.1$ \\
1 & 993 & 3 & 1125 & 1165 & 54.759 & 1219.759 & 127 & $* *$ \\
2 & 1037 & 1 & 1179.9 & 1219.9 & 54.759 & 1274.659 & 137.9 & $<0.1$ \\
3 & 1175 & 2 & 1234.8 & 1274.8 & 54.759 & 1329.559 & 54.8 & $<0.1$ \\
4 & 1219 & 1 & 1289.7 & 1329.7 & 39.071 & 1368.771 & 65.7 & $<0.1$ \\
5 & 1227 & 3 & 1328.9 & 1368.9 & 54.759 & 1423.659 & 96.9 & $<0.1$ \\
6 & 1307 & 2 & 1383.8 & 1423.8 & 54.759 & 1478.559 & 71.8 & $<0.1$ \\
7 & 1351 & 3 & 1438.7 & 1478.7 & 54.759 & 1533.459 & 82.7 & $<0.1$ \\
8 & 1389 & 1 & 1493.6 & 1533.6 & 55.942 & $\mathbf{1 5 8 9 . 5 4 2}$ & 99.6 & $<0.1$ \\
\hline
\end{tabular}

From Tables (13-15), the maximum completion times for the three CC devices are respectively equal to 1566.91, 1589.54 and 1470.58. The makespan $C_{\max }$ is then obtained for the value $1589.54 \mathrm{~min}$ of the production order. The sojourn time of this charge is equal to $99.6 \mathrm{~min}$ while the maximum allowed sojourn time is equal to $150 \mathrm{~min}$. The makespan corresponds to the charge number 8 of the sequence number 3 of the production order on $\mathrm{CC}_{2}$. The solution is computed within a CPU time of $1267 \mathrm{~s}$.

One can also remark that the continuity constraints are met for all the tests (col. 9) where the gap is less that 0.1 . This ensures computing feasible schedules for all the system.

In Table 16, column 1 represents the sequence number dedicated to a CC, column 2 represents the charge number $c$ of a sequence noted as a couple $(k, c)$, columns 3-5-6 represent the start times at each stage $\left(\mathrm{CV}_{i}-\mathrm{RS}_{j}\right.$ $\mathrm{CC}_{k}$ ) of a charge $c=1, \ldots, n_{k}$. Column 4 represents the $\mathrm{CV}$ number for which a charge $c$ is assigned to at the first stage. Column 7 represents the processing time computed for a charge at the last stage CC. Columns 8-9-10 represent respectively the completion time $\left(C_{\max }\right)$, the sojourn (transit) time and the continuity constrains for each charge. Column 11 represents the setup time between two successive sequences for the same CC device. Column 12 represents the dedicated CC machine to which a charge of a sequence is assigned. Moreover, we can remark that all the obtained continuity constraints are mostly met with an acceptable charges continuity.

The solutions are presented in Table 16 and the efficient makespan for the problem is equal to $C_{\max }=2605.966$ and was obtained for a total CPU of $1750 \mathrm{~s}$. The sojourn time of this charge is of $128 \mathrm{~min}$ where the maximum allowed sojourn time for the $\mathrm{CC}_{2}$ is equal to $150 \mathrm{~min}$. This efficient makespan is delivered for the processing termination time of the charge number 4 of the sequence number 2 dedicated to $\mathrm{CC}_{2}$ machine at the last stage.

In the Table 16, the RGA approach was running on a set of production orders with a 4 CC devices system at the last stage. 
TABle 15. A RGA results for $\mathrm{CC}_{3}$ for $\operatorname{SCC}([15,15,19] \times[7,10,8] \times[5,7,12])$.

\begin{tabular}{lllllllll}
\hline Charge $(k, c)$ & $x_{k, c}^{1}$ & $\mathrm{CV}_{i}$ & $x_{k, c}^{2}$ & $x_{k, c}^{3}$ & $p_{k, c}^{3} 3$ & Comp. time & Sojourn & cont. \\
\hline 1 & 7 & 3 & 77 & 118 & 53.2 & 171.2 & 59 & $* *$ \\
2 & 54 & 3 & 121 & 171.14 & 71.924 & 243.064 & 65.14 & $<0.1$ \\
3 & 136 & 1 & 203 & 243 & 73.08 & 316.08 & 55 & $<0.1$ \\
4 & 186 & 1 & 253 & 316.02 & 62.056 & 378.076 & 78.02 & $<0.1$ \\
5 & 230 & 1 & 338.01 & 378.01 & 62.056 & 440.066 & 96.01 & $<0.1$ \\
1 & 312 & 2 & 398 & 470.01 & 62.056 & 532.066 & 106.01 & $* *$ \\
2 & 370 & 2 & 461 & 532 & 44.062 & 576.062 & 110 & $<0.1$ \\
3 & 414 & 2 & 486 & 576 & 42.311 & 618.311 & 110 & $<0.1$ \\
4 & 488 & 1 & 574 & 618.25 & 40.283 & 658.533 & 78.25 & $<0.1$ \\
5 & 532 & 2 & 599 & 658.47 & 54.971 & 713.441 & 74.47 & $<0.1$ \\
6 & 590 & 1 & 673.38 & 713.38 & 54.971 & 768.351 & 71.38 & $<0.1$ \\
7 & 620 & 3 & 698.38 & 768.29 & 54.971 & 823.261 & 96.29 & $<0.1$ \\
1 & 714 & 3 & 813.2 & 853.2 & 54.971 & 908.171 & 87.2 & $* *$ \\
2 & 796 & 3 & 868.11 & 908.11 & 54.971 & 963.081 & 60.11 & $<0.1$ \\
3 & 846 & 1 & 923.01 & 963.01 & 39.283 & 1002.293 & 65.01 & $<0.1$ \\
4 & 854 & 1 & 962.23 & 1002.23 & 39.283 & 1041.513 & 96.23 & $<0.1$ \\
5 & 884 & 2 & 1001.46 & 1041.5 & 54.971 & 1096.471 & 105.5 & $<0.1$ \\
6 & 978 & 2 & 1045 & 1096.4 & 54.971 & 1151.371 & 66.4 & $<0.1$ \\
7 & 1030 & 1 & 1111.3 & 1151.3 & 54.971 & 1206.271 & 69.3 & $<0.1$ \\
8 & 1066 & 3 & 1166.2 & 1206.2 & 54.971 & 1261.171 & 88.2 & $<0.1$ \\
9 & 1110 & 2 & 1221.1 & 1261.1 & 54.971 & 1316.071 & 99.1 & $<0.1$ \\
10 & 1154 & 1 & 1276 & 1316 & 71.062 & 1387.062 & 110 & $<0.1$ \\
11 & 1280 & 1 & 1347 & 1387 & 44.364 & 1431.364 & 55 & $<0.1$ \\
12 & 1324 & 3 & 1391.3 & 1431.3 & 39.283 & 1470.583 & 55.3 & $<0.1$ \\
\hline
\end{tabular}

The numerical results detailed in Table 16 show that RGA obtains the solutions in less than $1 \mathrm{~s}$ for the first set of instances (small ones) (see Tab. 9). On the other hand, RGA computes the solution in a CPU time of $2105.31 \mathrm{~s}$ for instances that comprise up to 144 charges partitioned in 2 sequences and for a 2 CC system. However, for larger problems instances one should allow extra time to run the RGA and for big sets. For all these, there were no generated discontinuities (the gap between the end time and start time of a charge on a CC for two successive charges). We remark that there is no charge that has been exceeding the maximum allowed transit time $T_{k, c}$.

Table 17 shows that RGA succeed to obtain an efficient solution within a CPU time equal to $85.31 \mathrm{~s}$ for the case of a $2 \mathrm{CC}$ with 2 sequences of a total of 144 charges. While the algorithm was able to compute the solution within a CPU time of $1678.23 \mathrm{~s}$ for the case of a 3 sequences of a total of 100 charges for a $3 \mathrm{CC}$ system. Also, one can remark that the problem becomes big time consuming for the problems with more than 2 CC system.

Based on the tables above, we have shown the performance of the proposed RGA for several SCC instances and with different parameters.

We also have demonstrated that in the most cases the obtained solution gives the best trade-off between an acceptable running time, a minimal inter-sequence dependent setup times and minimal discontinuities. Also RGA ensures that in all cases, the maximum allowing sojourn time is respected.

\section{Conclusion}

This article considers a novel model to study a particular configuration of the SCC that is the inter-sequence dependent setup times SCC planning and scheduling problem. It is able to assign and sequence a big number of batches into several continuous casting devices. The problem can be seen as a particular case of the hybrid 
TABLE 16. A RGA results for a $4 \mathrm{CC}$ with large problem $\operatorname{SCC}([5,5,8] \times[6,4] \times[5,4,5] \times[6,6,7])$.

\begin{tabular}{|c|c|c|c|c|c|c|c|c|c|c|c|}
\hline Seq. $s_{k}$ & Charge $(k, c)$ & $x_{k, c}^{1}$ & $\mathrm{CV}_{i}$ & $x_{k, c}^{2}$ & $x_{k, c}^{3}$ & $p_{k, c}^{3}$ & Comp. time & Sojourn & Cont. & $\sigma_{s_{k}}$ & $\mathrm{CC}_{k}$ \\
\hline \multirow[t]{5}{*}{1} & 1 & 2 & 1 & 72 & 135 & 64.967 & 199.967 & 88 & & & 1 \\
\hline & 2 & 27 & 2 & 97 & 200 & 64.579 & 264.579 & 128 & 0.033 & & 1 \\
\hline & 3 & 132 & 1 & 202 & 265 & 64.589 & 329.589 & 88 & 0.421 & & 1 \\
\hline & 4 & 177 & 2 & 247 & 330 & 64.079 & 394.079 & 108 & 0.411 & & 1 \\
\hline & 5 & 222 & 3 & 292 & 485 & 64.546 & 639.546 & 128 & 90 & 90 & 1 \\
\hline \multirow[t]{5}{*}{2} & 1 & 327 & 2 & 397 & 550 & 64.888 & 614.888 & 88 & & & 1 \\
\hline & 2 & 392 & 2 & 462 & 615 & 64.489 & 679.489 & 88 & 0.112 & & 1 \\
\hline & 3 & 437 & 1 & 507 & 680 & 64.986 & 744.986 & 108 & 0.511 & & 1 \\
\hline & 4 & 487 & 3 & 557 & 745 & 64.996 & 809.996 & 123 & 0.014 & & 1 \\
\hline & 5 & 587 & 1 & 657 & 855 & 64.259 & 964.259 & 88 & 45 & 45 & 1 \\
\hline \multirow[t]{8}{*}{3} & 1 & 627 & 2 & 697 & 920 & 64.67 & 984.67 & 113 & & & 1 \\
\hline & 2 & 717 & 3 & 787 & 985 & 64.454 & 1049.454 & 88 & 0.33 & & 1 \\
\hline & 3 & 767 & 2 & 837 & 1050 & 64.375 & 1114.375 & 103 & 0.546 & & 1 \\
\hline & 4 & 807 & 3 & 877 & 1115 & 64.329 & 1179.329 & 128 & 0.625 & & 1 \\
\hline & 5 & 1002 & 2 & 1072 & 1180 & 95.982 & 1275.982 & 88 & 0.671 & & 1 \\
\hline & 6 & 1067 & 1 & 1137 & 1275 & 95.305 & 1370.305 & 118 & -0.982 & & 1 \\
\hline & 7 & 1157 & 3 & 1227 & 1370 & 95.448 & 1465.448 & 123 & -0.305 & & 1 \\
\hline & 8 & 1257 & 1 & 1327 & 1510 & 95.581 & 1650.581 & 118 & 45 & 45 & 1 \\
\hline \multirow[t]{6}{*}{1} & 1 & 1342 & 2 & 1412 & 1605 & 95.677 & 1700.677 & 128 & & & 2 \\
\hline & 2 & 1437 & 2 & 1507 & 1700 & 95.156 & 1795.156 & 128 & -0.677 & & 2 \\
\hline & 3 & 1632 & 1 & 1702 & 1795 & 85.985 & 1880.985 & 88 & -0.156 & & 2 \\
\hline & 4 & 1677 & 3 & 1747 & 1880 & 85.863 & 1965.863 & 128 & -0.985 & & 2 \\
\hline & 5 & 1782 & 3 & 1852 & 1965 & 85.994 & 2050.994 & 108 & -0.863 & & 2 \\
\hline & 6 & 1882 & 2 & 1952 & 2110 & 85.334 & 2255.334 & 93 & 59.006 & 60 & 2 \\
\hline \multirow[t]{4}{*}{2} & 1 & 1937 & 2 & 2007 & 2195 & 85.932 & 2280.932 & 123 & & & 2 \\
\hline & 2 & 2017 & 1 & 2087 & 2280 & 85.721 & 2365.721 & 128 & -0.932 & & 2 \\
\hline & 3 & 2142 & 1 & 2212 & 2365 & 85.942 & 2450.942 & 88 & -0.721 & & 2 \\
\hline & 4 & 2187 & 2 & 2257 & 2485 & 85.966 & 2605.966 & 128 & 34.058 & 35 & 2 \\
\hline \multirow[t]{5}{*}{1} & 1 & 132 & 1 & 202 & 305 & 50.188 & 355.188 & 128 & & & 3 \\
\hline & 2 & 182 & 3 & 252 & 355 & 50.392 & 405.392 & 128 & -0.188 & & 3 \\
\hline & 3 & 237 & 3 & 307 & 405 & 50.492 & 455.492 & 123 & -0.392 & & 3 \\
\hline & 4 & 282 & 1 & 352 & 455 & 50.652 & 505.652 & 128 & -0.492 & & 3 \\
\hline & 5 & 332 & 1 & 402 & 550 & 50.422 & 600.422 & 128 & 44.348 & 45 & 3 \\
\hline \multirow[t]{4}{*}{2} & 1 & 482 & 3 & 552 & 600 & 50.019 & 650.019 & 88 & & & 3 \\
\hline & 2 & 532 & 2 & 602 & 650 & 50.223 & 700.223 & 88 & -0.019 & & 3 \\
\hline & 3 & 582 & 2 & 652 & 700 & 50.282 & 750.282 & 88 & -0.223 & & 3 \\
\hline & 4 & 632 & 1 & 702 & 785 & 50.93 & 940.93 & 88 & 0.718 & 35 & 3 \\
\hline \multirow[t]{5}{*}{3} & 1 & 672 & 1 & 742 & 802 & 50.417 & 955.417 & 98 & & & 3 \\
\hline & 2 & 752 & 1 & 822 & 852 & 60.915 & 1015.915 & 128 & -0.417 & & 3 \\
\hline & 3 & 812 & 2 & 882 & 912 & 60.594 & 1075.594 & 128 & -0.915 & & 3 \\
\hline & 4 & 912 & 1 & 982 & 972 & 60.251 & 1135.251 & 88 & -0.594 & & 3 \\
\hline & 5 & 932 & 2 & 1002 & 1062 & 60.511 & 1255.511 & 128 & 29.749 & 30 & 3 \\
\hline \multirow[t]{6}{*}{1} & 1 & 992 & 3 & 1062 & 1122 & 60.875 & 1285.875 & 128 & & & 4 \\
\hline & 2 & 1092 & 2 & 1162 & 1182 & 60.639 & 1345.639 & 88 & -0.875 & & 4 \\
\hline & 3 & 1112 & 2 & 1182 & 1242 & 60.389 & 1405.389 & 128 & -0.639 & & 4 \\
\hline & 4 & 1212 & 2 & 1282 & 1302 & 60.414 & 1465.414 & 88 & -0.389 & & 4 \\
\hline & 5 & 1292 & 2 & 1362 & 1362 & 50.684 & 1515.684 & 128 & -0.414 & & 4 \\
\hline & 6 & 1342 & 3 & 1412 & 1472 & 50.426 & 1685.426 & 128 & 59.316 & 60 & 4 \\
\hline
\end{tabular}


TABLE 16. (Continued)

\begin{tabular}{|c|c|c|c|c|c|c|c|c|c|c|c|}
\hline Seq. $s_{k}$ & Charge $(k, c)$ & $x_{k, c}^{1}$ & $\mathrm{CV}_{i}$ & $x_{k, c}^{2}$ & $x_{k, c}^{3}$ & $p_{k, c}^{3}$ & Comp. time & Sojourn & Cont. & $\sigma_{s_{k}}$ & $\mathrm{CC}_{k}$ \\
\hline \multirow[t]{6}{*}{2} & 1 & 1392 & 1 & 1462 & 1522 & 50.074 & 1675.074 & 128 & & & 4 \\
\hline & 2 & 1482 & 3 & 1552 & 1572 & 50.343 & 1725.343 & 88 & -0.074 & & 4 \\
\hline & 3 & 1492 & 1 & 1562 & 1622 & 50.834 & 1775.834 & 128 & -0.343 & & 4 \\
\hline & 4 & 1542 & 3 & 1612 & 1672 & 50.354 & 1825.354 & 128 & -0.834 & & 4 \\
\hline & 5 & 1677 & 1 & 1762 & 1722 & 55.11 & 1880.11 & 103 & -0.354 & & 4 \\
\hline & 6 & 1747 & 1 & 1817 & 1807 & 55.522 & 1995.522 & 88 & 29.89 & 30 & 4 \\
\hline \multirow[t]{7}{*}{3} & 1 & 1802 & 2 & 1872 & 1862 & 55.24 & 2020.24 & 88 & & & 4 \\
\hline & 2 & 1827 & 3 & 1927 & 1917 & 55.068 & 2075.068 & 118 & -0.24 & & 4 \\
\hline & 3 & 1872 & 1 & 1942 & 1972 & 55.866 & 2130.866 & 128 & -0.068 & & 4 \\
\hline & 4 & 1927 & 3 & 1997 & 2027 & 55.6 & 2185.6 & 128 & -0.866 & & 4 \\
\hline & 5 & 1982 & 3 & 2052 & 2082 & 55.026 & 2240.026 & 128 & -0.6 & & 4 \\
\hline & 6 & 2037 & 3 & 2117 & 2137 & 55.306 & 2295.306 & 128 & -0.026 & & 4 \\
\hline & 7 & 2092 & 1 & 2162 & 2192 & 55.754 & 2350.754 & 128 & -0.306 & & 4 \\
\hline
\end{tabular}

TABLE 17. RGA experiment results for different CC systems.

\begin{tabular}{lllll}
\hline \# Inst. & Batch & Best $C_{\max }$ & CPU $(\mathrm{sec})$ & Tot. charges \\
\hline 1 & $(5) \times(5) \times(5)$ & 427.58 & 1.09 & 15 \\
2 & $(14,21) \times(5,5,5)$ & 1225.15 & 2.12 & 50 \\
3 & $(15,10,12) \times(5,5,5)$ & 1559.00 & 5.75 & 52 \\
4 & $(15,14,30) \times(5,5,5)$ & 1807.01 & 6.55 & 74 \\
5 & $(10,13,17,8) \times(5,5,5,5,5)$ & 1756.72 & 40.99 & 73 \\
6 & $(18,11,23,15,27) \times(5,5,5,5,5,5,5,5,5,5)$ & 2506,04 & 85.31 & 144 \\
7 & $(10,10) \times(10,10) \times(10,10)$ & 706.07 & 7.45 & 60 \\
8 & $(15,15,19) \times(7,10,8) \times(5,7,12)$ & 1589.54 & 8.31 & 98 \\
9 & $(5,15,5,10) \times(10,10,10,5) \times(5,5,5,5)$ & 1239.34 & 67.23 & 90 \\
10 & $(5,10,5,10,5) \times(10,10,10,5,5) \times(5,5,5,5,5)$ & 1678.23 & 217.12 & 100 \\
11 & $(5,5,5) \times(6,4) \times(5,4,5) \times(6,6,7)$ & 2605.96 & 90.86 & 58 \\
12 & $(10,10,10,10) \times(10,10,10) \times,(5,5,5) \times(5,5)$ & 1543.27 & 387.12 & 95 \\
13 & $(5,5,5,5,5) \times(10,5,10,5) \times(5,5,5) \times(5,5) \times(10,5)$ & 1278.65 & 567.35 & 95 \\
\hline
\end{tabular}

flowshop problem (HFS) with 3 stages which is also known as the flexible flowshop problem. The objective stated as the Makespan is to minimize the maximum completion time of the last charge of the last sequence while considering the inter-sequence dependent setup times between sequences at the last stage. We developed a generalized model that could consider any number of the underlying devices at each stage and for any number of the dedicated sequences. One of the remarks that we can make is that the studied problem is very difficult and handles a huge number of decision variables that make it intractable for big size instances with a production bottleneck at the last stage.

The proposed RGA approach provides suitable operations to avoid infeasible solutions by including two informations in each gene $(k, c)$ that are the controlling and coding operations. The first information controls coding of the genes. The second information contains the genetic information. These strategies show the flexibility and ability to produce efficient solutions for the studied problem.

We have recorded some promising results by considering setup times that exist between sequences. In fact, setup times are only to take into account for the last charge for a sequence and the first charge of the next sequence to process on the same CC device. We have ensured that: (i) the sojourn time must be satisfied, (ii) the setup times must be minimized and (iii) the continuity constraints must be satisfied. The RGA approach 
that we developed gives satisfactory results in terms of the number of the processed sequences with different sizes and densities. Our approach were specifically tailored for the studied problem with specific chromosomes encoding and specific evolutionary operators (crossover, mutation,...). The algorithm was set to run on both real life similar problems and other randomly generated ones to establish the limits of the run time.

To overcome the complexity and the hardness of the SCC, some future research may consider to embed an high performance computing based GPU to minimize the total run time to be able to solve very large instances for the SCC industrial system.

\section{REFERENCES}

[1] A. Atighehchian, M. Bijari and H. Tarkesh, A novel hybrid algorithm for scheduling steelmaking continuous casting production. Comput. Oper. Res. 36 (2009) 2450-246.

[2] S. Basu and G. Dutta, A Survey of the Non-Optimization techniques used in an integrated steel plant. Manag. Dyn. 6 (2006) $33-68$.

[3] A. Bellabdaoui and J. Teghem, A mixed-integer linear programming model for the continuous casting planning. Int. J. Prod. Econom. 104 (2006) 260-270.

[4] A. Bellabdaoui, A. Fiordaliso and J. Teghem, A heuristic algorithm for scheduling the steelmaking continuous casting process. Pac. J. Optim. 1 (2005) 447-464.

[5] J. Blazewicz, K. Ecker, E. Pesch, G. Schmidt and J. Weglarz, Scheduling Computer Manufacturing Processes. Springer (1996).

[6] N. Chakraborti, R. Kumar and D. Jain, A study of the continuous casting mold using a pareto-converging genetic algorithm. Appl. Math. Model. 25 (2001) 287-297.

[7] P.C. Chang and S.H. Chen, Integrating Dominance Properties with Genetic Algorithms for Parallel Machine Scheduling Problems with Setup Times. Appl. Soft Comput. 11 (2011) 1263-1274.

[8] L. Chen, N. Bostel, P. Dejax, J.C. Cai and L.F. Xi, A tabu search algorithm for the integrated scheduling problem of container handling systems in a maritime terminal. Eur. J. Oper. Res. 181 (2007) 40-58.

[9] P.I. Cowling, D. Ouelhadj and S. Petrovic, Dynamic scheduling of steel casting and mill using multi-agents. Prod. Plan. Control 15 (2004) 495-501.

[10] B. de Schutter, Designing optimal timing and sequencing strategies for a continuous steel foundry, in Proceedings of the European Control Conference 1999 (ECC'99), Karlsruhe, Germany, Paper 160/BP-2.6, Aug.-Sept. (1999).

[11] G. Dutta and R. Fourer, A survey of mathematical programming application in integrated steel plants. Manuf. Service Oper. Manag. 3 (2001) 387-400.

[12] I. Ferretti, S. Zanoni and L. Zavanella, Production-inventory scheduling using ant system metaheuristic. Int. J. Prod. Econom. 104 (2008) 317-326.

[13] M. Garey and D. Johnson, Computers and Intractability: A Guide to the Theory of Np-Completness. W.H. Freeman and Company, San Francisco (1979).

[14] J.N.D. Gupta, Two-stage, hybrid flowshop scheduling problem. J. Oper. Res. Soc. 39 (1988) 359-364.

[15] I. Harjunkoski and I.E. Grossmann, A decomposition approach for the scheduling of a steel plant production. Comput. Chem. Eng. 25 (2001) 1647-1660.

[16] M. Helal, G. Rabadi and A. Al-Salem, A tabu search algorithm to minimize the makespan for the unrelated parallel machines scheduling problem with setup times. Int. J. Oper. Res. 3 (2006) 182-192.

[17] J.R. Kalagnanam, M.W. Dawande, M. Trumbo and H.S. Lee, The surplus inventory matching problem in the process industry. Oper. Res. 48 (2000) 505-516.

[18] C.-H. Ko and S.-F. Wang, Precast production scheduling using multi-objective genetic algorithms. Expert Syst. Appl. 38 (2011) 8293-8302.

[19] H.S. Lee, S.S. Murthy, S.W. Haider and D.V. Morse, Primary production scheduling at steelmaking industries. IBM J. Res. Develop 40 (1996) 231-252.

[20] K. Lee, S.Y. Chang and Y. Hong, Continuous slab caster scheduling and interval graphs. Prod. Plan. Control 15 (2004) 495-501.

[21] L. Li, Q. Tang, P. Peng Zheng, L. Zhang and C.A. Floudas, An improved self-adaptive genetic algorithm for scheduling steelmaking continuous casting production, in Proceedings of the 6th International Asia Conference on Industrial Engineering and Management Innovation (IEMI2015), Core Theory and Applications of Industrial Engineering, 1: 399-410, Tianjin, July 25-26th (2015).

[22] R. Linn and W. Zhang, Hybrid flow shop scheduling: a survey. Comput. Ind. Eng. 31 (1999) 57-61.

[23] H. Missbauer, W. Hauber and W. Stadler, A scheduling system for the steelmaking-continuous casting process. A case study from the steel-making industry. Int. J. Prod. Res. 47 (2009) 4147-4172.

[24] B. Naderi, M. Zandieh, A.K.G. Balagh and V. Roshanaei, An improved simulated annealing for hybrid flowshops with sequencedependent setup and transportation times to minimize total completion time and total tardiness. Expert Syst. Appl. 36 (2009) 9625-9633.

[25] T. Nishi, Y. Hiranaka and M. Inuiguchi, Lagrangian relaxation with cut generation for hybrid flow shop scheduling problems to minimize the total weighted tardiness. Comput. Oper. Res. 37 (2010) 189-198. 
[26] D. Pacciarelli and M. Pranzo, Production scheduling in a steelmaking-continuous casting plant. Comput. Chem. Eng. 28 (2004) 2823-2835.

[27] Q.K. Pan, An effective co-evolutionary artificial bee colony algorithm for steelmaking-continuous casting scheduling. Eur. J. Oper. Res. 250 (2016) 702-714.

[28] Q.K. Pan, L. Wang, K. Mao, J.H. Zhao and M. Zhang, An effective artificial bee colony algorithm for a real-world hybrid flowshop problem in steelmaking process. IEEE Trans. Autom. Sci. Eng. 10 (2013) 307-322.

[29] C. Rajendran and D. Chaudhuri, A multi-stage parallel processor flowshop problem with minimum flowtime. Eur. J. Oper. Res. 57 (1992) 11-122.

[30] R. Ruiz and C. Maroto, A genetic algorithm for hybrid flowshops with sequence dependent setup times and machine eligibility. Eur. J. Oper. Res. 169 (2006) 781-800.

[31] A. Sbihi, A. Bellabdaoui and J. Teghem, Solving a mixed integer linear program with times setup for the steel-continuous casting planning and scheduling problem. Int. J. Prod. Res. 52 (2014) 7276-7296.

[32] H. Sherali, S. Sarin and M. Kodialam, Models and algorithms for a two-stage production process. Prod. Plan. Control 1 (1990) $27-39$.

[33] D.F. Shiau, S.C. Cheng and Y.M. Huang, Proportionate flexible flow shop scheduling via a hybrid constructive genetic algorithm. Expert Syst. Appl. 34 (2008) 1133-1143.

[34] L. Tang, and G. Wang, Decision Support system for the batching problems of steelmaking and continuous-casting production. Omega Int. J. Manag. Sci. 36 (2008) 976-991.

[35] L. Tang, J. Liu, A. Rong and Z. Yang, A mathematical programming model for scheduling steelmaking-continuous casting production. Eur. J. Oper. Res. 120 (2000) 423-435.

[36] L. Tang, J. Liu, A. Rong and Z. Yang, A review of planning and scheduling systems and methods for integrated steel production. Eur. J. Oper. Res. 133 (2001) 1-20.

[37] L. Tang, P.B. Luh, J. Liu and L. Fang, Steel-making process scheduling using Lagrangian relaxation. Int. J. Prod. Res. 40 (2002) 55-70.

[38] L. Tang, H. Xuan and J. Liu, A new lagrangian relaxation algorithm for hybrid flow shop scheduling to minimize total weighted completion time. Comput. Oper. Res. 33 (2006) 3344-3359.

[39] L. Tang, X. Wang and J. Liu, Color-coating production scheduling for coils in inventory in steel industry. Autom. Sci. Eng. IEEE Trans. 5 (2008) 544-549.

[40] W.S. Um, Computer simulation of the steelmaking process with ARENA. J. Korean Soc. Maint. Eng. 7 (2002) $77-90$.

[41] H. Xuan and L. Tang, Scheduling a hybrid flow shop with batch production at the last stage. Comput. Oper. Res. 34 (2007) $2178-2733$.

[42] J. Yang, H. Che, F.P. Dou and T. Zhou, Genetic algorithm-based optimization used in rolling schedule. J. Iron Steel Res. Int. $5(2008)$ 18-22.

[43] V. Yaurima, L. Burtseva and A. Tchernykh, Hybrid flowshop with unrelated machines, sequence dependent setup time, availability constraints and limited buffers. Comput. Ind. Eng. 56 (2009) 1452-1463.

[44] D.F. Zhu, Z. Zheng and X.Q. Gao, Intelligent optimization-based production planning and simulation analysis for steelmaking and continuous casting process. J. Iron Steel Res. Int. 17 (2010) 19-24. 